\title{
A mathematical and numerical framework for bubble meta-screens*
}

\author{
Habib Ammari ${ }^{\dagger} \quad$ Brian Fitzpatrick $^{\dagger} \quad$ David Gontier ${ }^{\dagger} \quad$ Hyundae Lee $^{\ddagger}$ \\ Hai Zhang§
}

\begin{abstract}
The aim of this paper is to provide a mathematical and numerical framework for the analysis and design of bubble meta-screens. An acoustic meta-screen is a thin sheet with patterned subwavelength structures, which nevertheless has a macroscopic effect on the acoustic wave propagation. In this paper, periodic subwavelength bubbles mounted on a reflective surface (with Dirichlet boundary condition) is considered. It is shown that the structure behaves as an equivalent surface with Neumann boundary condition at the Minnaert resonant frequency which corresponds to a wavelength much greater than the size of the bubbles. Analytical formula for this resonance is derived. Numerical simulations confirm its accuracy and show how it depends on the ratio between the periodicity of the lattice, the size of the bubble, and the distance from the reflective surface. The results of this paper formally explain the super-absorption behavior observed in [V. Leroy et al., Phys. Rev. B, 2015].
\end{abstract}

Mathematics Subject Classification (MSC2000): 35R30, 35C20.

Keywords: Minnaert resonance, array of bubbles, periodic Green's function, metasurfaces.

\section{Introduction}

In this article we study the reflection properties of a meta-screen from a mathematical point of view. Broadly speaking, a meta-screen is a thin sheet with patterned subwavelength structures which has a macroscopic effect on the reflection and transmission of waves.

One way to design meta-screens is to put microscopic gas inclusions along a periodic lattice. The properties of such screens have been studied in recent years, with spectacular results. In [22, 25], it was experimentally shown how the reflection and transmission coefficients vary with respect to the wavelength of the incoming acoustic wave. It was later shown how super-absorption may be achieved with these meta-screens [24], i.e. null reflection and null transmission coefficients.

\footnotetext{
${ }^{*}$ Hyundae Lee was supported by NRF-2015R1D1A1A01059357 grant. Hai Zhang was supported by the initiation grant IGN15SC05 from HKUST.

${ }^{\dagger}$ Department of Mathematics, ETH Zürich, Rämistrasse 101, CH-8092 Zürich, Switzerland (habib.ammari@math.ethz.ch, brian.fitzpatrick@sam.math.ethz.ch, david.gontier@sam.math.ethz.ch ).

${ }^{\ddagger}$ Department of Mathematics, Inha University, 253 Yonghyun-dong Nam-gu, Incheon 402-751, Korea (hdlee@inha.ac.kr).

${ }^{\S}$ Department of Mathematics, HKUST, Clear Water Bay, Kowloon, Hong Kong (haizhang@ust.hk).
} 
These phenomena can be explained by the use of subwavelength resonators in the design of the meta-screens. In [9] for instance, a mathematical justification was given when the meta-screen was made of subwavelength plasmonic particles, where resonance is due to negative dielectric coefficient [7]. Minnaert bubbles act like plasmonic nanoparticles. We refer the reader to $[7,8,10,11,20]$ for the mathematical analysis of resonances for plasmonic nanoparticles.

In this paper, we study the case where the resonance is due to the high contrast in density between the gas inclusions and the surrounding medium, characterized by a small parameter $\delta$ representing the inverse of the contrast. This resonance, known as the Minnaert resonance, was observed and explained as early as 1933 [27] (see also [23, 17]). We recently gave a rigorous mathematical justification of this resonance in the case of a single bubble in a homogeneous medium [5]. We studied the acoustic property of the bubble, and proved that Minnaert resonance occurred when the frequency is appropriately propositional to $\frac{\sqrt{\delta}}{s}$ with $s$ being the size of the bubble.

In the present paper, we use similar techniques as in [9] to study the reflection properties of a meta-screen when both the typical size of the periodic cell and the size of the bubbles are subwavelength, and the contrast $\delta^{-1}$ is large. We also investigate the limiting case when $a$ goes to 0 and $\delta$ goes to 0 proportional to $a^{2}$. Note that in the case where $\delta$ is a fixed parameter, the usual homogenization techniques can be applied, and a large literature already exists on describing boundary layer effects $[1,2,3]$. In our case, because of the excitation of resonace, one must resort to other methods. The idea of using an unbounded parameter as the size of the cells goes to zero is not new, and is sometimes referred to as «high-contrast homogenization» $[13,14,19]$. This technique has already been successful in explaining some spectacular physical phenomena [12].

Our main result is the following. We consider a periodic subwavelength bubbles above a reflective surface ( with Dirichlet boundary condition). Then, under the appropriate scaling $\delta=\mu a^{2}$, Minnaert resonance can be excited at a fixed frequency of order one, and the surface behaves as an equivalent surface with Neumann boundary condition at this frequency at the limit $a \rightarrow 0$. The theorem is valid for all shapes of Minnaert resonators. When taking into account some extra physical damping effects that do not appear in our mathematical model [21], this eventually explains the super-absorption behavior witnessed in [24] (see Remark 2.4).

The paper is structured as follows. In Section 2, we fix the notations of the experiment under consideration, and state our main result, the proof of which is detailed in Section 3. The proof uses layer potential techniques and asymptotic expansions. Finally, numerical results are presented in Section 4.

\section{Statement of the problem}

We consider a reflective surface, on top of which small scatterers are arranged along some periodic lattice. A typical example of such a situation is given by air bubbles arranged in water, as described in [24, 25].

Let us fix some notation. We will state our results in dimension $d \in\{2,3\}$, and write $\mathbb{R}^{d} \ni \mathbf{x}=\left(\bar{x}, x_{d}\right)$, with $\bar{x} \in \mathbb{R}^{d-1}$ and $x_{d} \in \mathbb{R}$. We let $\partial \mathbb{R}_{+}^{d}:=\left\{\mathbf{x} \in \mathbb{R}^{d}, x_{d}=0\right\}$ represent the reflective plane, and $\mathbb{R}_{ \pm}^{d}:=\left\{\mathbf{x} \in \mathbb{R}^{d}, \pm x_{d}>0\right)$ be the upper and lower half space. The shape of 
the bubbles is described by a simply connected domain $D \subset \mathbb{R}_{+}^{d}$ with smooth boundary $\partial D$. The bubbles are arranged periodically along a lattice $\mathcal{R}$ of $\mathbb{R}^{d-1}$. For instance, if $d=2$, then $\mathcal{R}=a \mathbb{Z}$ for some $a>0$.

For $\varepsilon>0$, we denote by $\Omega^{\varepsilon}$ the volume occupied by the bubbles. More specifically, we set

$$
\Omega^{\varepsilon}:=\bigcup_{\mathbf{R} \in \mathcal{R}} \varepsilon(D+\mathbf{R})
$$

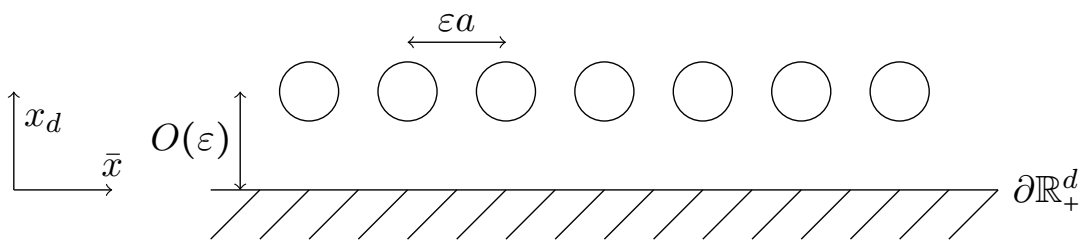

We denote by $\rho_{b}$ and $\kappa_{b}$ the density and bulk modulus of the air inside the bubbles, and by $\rho$ and $\kappa$ the corresponding parameters for the background medium $\mathbb{R}^{d} \backslash \overline{\Omega^{\varepsilon}}$. We consider the scattering of acoustic waves by this meta-screen. In the sequel, $\omega /(2 \pi)$ represents the frequency of the source, and

$$
v=\sqrt{\frac{\lambda}{\rho}}, \quad v_{b}=\sqrt{\frac{\lambda_{b}}{\rho_{b}}}, \quad k=\frac{\omega}{v}, \quad \text { and } \quad k_{b}=\frac{\omega}{v_{b}}
$$

denote respectively the speed of sound outside and inside the bubbles, and the wave number outside and inside the bubbles. Finally, we introduce the dimensionless contrast parameter

$$
\delta=\frac{\rho_{b}}{\rho} .
$$

We assume that $\delta \ll 1$. With appropriate physical units, we also assume that $k=O(1)$, $k_{b}=O(1)$, and the size of $D$ is also of order one. With these in mind, the acoustic problem is (we use capital letters for macroscopic fields)

$$
\left\{\begin{array}{l}
\left(\Delta+k^{2}\right) U^{\varepsilon}=0 \text { on } \mathbb{R}_{+}^{d} \backslash \overline{\Omega^{\varepsilon}} \\
\left(\Delta+k_{b}^{2}\right) U^{\varepsilon}=0 \text { on } \Omega^{\varepsilon} \\
\left.U^{\varepsilon}\right|_{+}=\left.U^{\varepsilon}\right|_{-} \text {on } \partial \Omega^{\varepsilon} \\
\left.\partial_{\nu} U^{\varepsilon}\right|_{-}=\left.\delta \partial_{\nu} U^{\varepsilon}\right|_{+} \text {on } \partial \Omega^{\varepsilon} \\
U^{s}:=U^{\varepsilon}-U^{\text {in }} \text { satisfies the outgoing radiation condition, } \\
U^{\varepsilon}=0 \text { on } \partial \mathbb{R}_{+}^{d}
\end{array}\right.
$$

where $U^{\text {in }}$ is some incoming pressure wave satisfying $\left(\Delta+k^{2}\right) U^{\text {in }}=0$ and $\cdot_{ \pm}$denotes the limits from respectively outside and inside of $\Omega^{\varepsilon}$. In this paper, we consider the special case where the incoming pressure $U^{\text {in }}$ is a plane wave going towards the plane from the upper half-space, so we set

$$
U_{\mathbf{k}}^{\mathrm{in}}(\mathbf{X})=u_{0} \mathrm{e}^{-\mathrm{i} \mathbf{k} \cdot \mathbf{X}}=u_{0} \mathrm{e}^{-\mathrm{i} \bar{k} \cdot \bar{X}} \mathrm{e}^{-\mathrm{i} k_{d} X_{d}},
$$

where $\mathbf{k}=\left(\bar{k}, k_{d}\right) \in \mathbb{R}^{d-1} \times \mathbb{R}$, with $k_{d}>0$ and $|\mathbf{k}|=k$, is the wave vector. 
Such problems have been extensively studied using homogenization theory in recent decades. For instance, the scattered field is well-understood as $\varepsilon \rightarrow 0$, and is of order $\varepsilon[2,3,4]$. In the present article, we study the special case where the contrast $\delta$ is also scaled with $\varepsilon \rightarrow 0$. As we will see, such a regime, which is accessible physically, presents some interesting features.

More specifically, according to [5], there is a resonance phenomenon in the regime $\sqrt{\delta} \sim \varepsilon$ (Minnaert resonance). In the sequel, we fix $\mu>0$, and study (2.1), with

$$
\delta:=\delta_{\varepsilon}=\mu \varepsilon^{2} .
$$

In this case, standard homogenization techniques are no longer applicable and new techniques are needed.

In the absence of bubbles, the solution of (2.1) is simply

$$
U_{0}(\mathbf{X}):=U_{\mathbf{k}}^{\mathrm{in}}\left(\bar{X}, X_{d}\right)-U_{\mathbf{k}}^{\mathrm{in}}\left(\bar{X},-X_{d}\right)=-2 \mathrm{i} u_{0} \mathrm{e}^{-\mathrm{i} \bar{k} \cdot \bar{X}} \sin \left(k_{d} X_{d}\right) .
$$

In the presence of bubbles, we expect this solution to be perturbed. Our goal is to describe the main contribution of this perturbation. In order to state our results, we introduce some extra-notation. First, we introduce a constant $\mu_{M}$ defined by

$$
\mu_{M}:=\frac{k_{b}^{2}|D|}{C_{D, \mathcal{R}}^{+}},
$$

where $|D|$ is the volume of $D$, and $C_{D, \mathcal{R}}^{+}$is the periodic capacity defined in Definition 3.6. Then we introduce the scattering function

$$
g_{s}(\mu, \varepsilon):=\frac{\varepsilon M_{1}}{1-\frac{\mu_{M}}{\mu}-\varepsilon \mathrm{i} \frac{M_{1}^{2} k_{d} C_{D, \mathcal{R}}^{+}}{|\Gamma|}},
$$

where the constant $M_{1}$ is defined in (3.17) below and $|\Gamma|$ is the volume of the fundamental cell of $\mathcal{R}$. We also introduce the functions $\alpha_{0}$ and $\alpha_{1}: \alpha_{0}$ is the (unique) solution to the problem

$$
\left\{\begin{array}{l}
\Delta \alpha_{0}=0 \text { on } \mathbb{R}_{+}^{d} \backslash \overline{\Omega^{1}}, \\
\left.\alpha_{0}\right|_{+}=1 \text { on } \partial \Omega^{1}, \\
\alpha_{0}-\alpha_{0, \infty} \text { is exponentially decaying as } x_{d} \rightarrow \infty \\
\alpha_{0}=0 \text { on } \partial \mathbb{R}_{+}^{d},
\end{array}\right.
$$

and $\alpha_{1}$ is the (unique) solution to the problem

$$
\left\{\begin{array}{l}
\Delta \alpha_{1}=0 \text { on } \mathbb{R}_{+}^{d} \backslash \overline{\Omega^{1}}, \\
\left.\alpha_{1}\right|_{+}=\left.x_{d}\right|_{+} \text {on } \partial \Omega^{1}, \\
\alpha_{1}-\alpha_{1, \infty} \text { is exponentially decaying as } x_{d} \rightarrow \infty, \\
\alpha_{1}=0 \text { on } \partial \mathbb{R}_{+}^{d} .
\end{array}\right.
$$

The exact values of $\alpha_{0, \infty}$ and $\alpha_{1, \infty}$ are given in Lemma 3.11 below. The function $\alpha_{0}$ is related to the monopole moment of bubbles, while $\alpha_{1}$ is related to their dipole moment. 
Finally, we introduce a functional space. Let $L \in \mathbb{R}^{+}$be large enough so that for all $\mathbf{y} \in D$, it holds that $y_{d} \leq L$ (hence $\left.0 \leq y_{d} \leq L\right)$. For $a \in \mathbb{R}^{+}$, we denote by $S_{a}:=\mathbb{R}^{d-1} \times(a, \infty)$, and we denote by $W^{1, \infty}\left(S_{a}\right)$ the usual Sobolev space with norm

$$
\|f\|_{W^{1, \infty}\left(S_{a}\right)}:=\sup _{\mathbf{X} \in S_{a}}|f|(\mathbf{X})+\sup _{\mathbf{X} \in S_{a}}|\nabla f|(\mathbf{X}) .
$$

Our main result is the following.

Theorem 2.1. There exists $C \in \mathbb{R}^{+}$such that, for all $\varepsilon \geq 0$ and all $\mu>0$, it holds that

$$
\left\|U^{\varepsilon}(\mathbf{X})-U_{0}(\mathbf{X})-\left(U_{1}^{\varepsilon}(\mathbf{X})+U_{\mathrm{BL}}^{\varepsilon}\left(\mathbf{X}, \frac{\mathbf{X}}{\varepsilon}\right)\right)\right\|_{W^{1, \infty}\left(S_{\varepsilon L}\right)} \leq C \varepsilon\left|g_{s}(\mu, \varepsilon)\right|,
$$

where

$$
\begin{aligned}
U_{\mathrm{BL}}^{\varepsilon}(\mathbf{X}, \mathbf{x}) & =\left(2 \mathrm{i} u_{0} k_{d}\right) \mathrm{e}^{-\mathrm{i} \bar{k} \cdot \bar{X}}\left[\varepsilon\left(\alpha_{1}(\mathbf{x})-\alpha_{1, \infty}\right)-g_{s}(\mu, \varepsilon)\left(\alpha_{0}(\mathbf{x})-\alpha_{0, \infty}\right)\right], \\
U_{1}^{\varepsilon}(\mathbf{X}) & =\left(2 \mathrm{i} u_{0} k_{d}\right) \mathrm{e}^{\mathrm{i}\left(k_{d} X_{d}-\bar{k} \cdot \bar{X}\right)}\left(\varepsilon \alpha_{1, \infty}-g_{s}(\mu, \varepsilon) \alpha_{0, \infty}\right),
\end{aligned}
$$

and $g_{s}(\mu, \varepsilon)$ is defined by (2.3). Moreover, $U_{\mathrm{BL}}^{\varepsilon}$ is exponentially decaying as $x_{d} \rightarrow \infty$.

The proof of the above theorem will be given in Section 3. The function $U_{\mathrm{BL}}^{\varepsilon}$ describes the behavior of the field near the bubbles (at distances of order $O(\varepsilon)$ ), while the function $U_{1}^{\varepsilon}$ describes the far field. Both functions have an $O(\varepsilon)$ part due to the dipole moment, and a resonant part $g_{s}(\mu, \varepsilon)$ due to the monopole moment.

In particular, from the behavior of $U^{\varepsilon}$ in the far-field, namely

$$
U^{\varepsilon}(\mathbf{X}) \approx u_{0} \mathrm{e}^{-\mathrm{i}\left(k_{d} X_{d}+\bar{k} \cdot \bar{X}\right)}+u_{0}\left(-1+2 \mathrm{i} k_{d}\left(\varepsilon \alpha_{1, \infty}-g_{s}(\mu, \varepsilon) \alpha_{0, \infty}\right)\right) \mathrm{e}^{\mathrm{i}\left(k_{d} X_{d}-\bar{k} \cdot \bar{X}\right)},
$$

we directly read the reflection coefficient

$$
R_{\varepsilon}=-1+2 \mathrm{i} k_{d}\left(\varepsilon \alpha_{1, \infty}-g_{s}(\mu, \varepsilon) \alpha_{0, \infty}\right) .
$$

As a consequence, we obtain

Theorem 2.2. In the case when $\mu=\mu_{M}$, in the limit as $\varepsilon \rightarrow 0$, we get

$$
R_{\varepsilon}=-1+2=1 .
$$

Therefore the equivalent screen has Neumann boundary condition for the wave equation.

Remark 2.3. If we neglect the effect of $\alpha_{1, \infty}$ for simplicity, then

$$
R_{\varepsilon} \approx-1-2\left(\frac{\varepsilon \mathrm{i} k_{d} M_{1}^{2} C_{D, \mathcal{R}}^{+}|\Gamma|^{-1}}{1-\frac{\mu_{M}}{\mu}-\mathrm{i} \varepsilon \frac{k_{d} M_{1}^{2} C_{D, \mathcal{R}}^{+}}{|\Gamma|}}\right) .
$$

Using the frequency variable $\omega$, we see that

$$
R(\omega) \approx-1-2\left(\frac{\mathrm{i} \frac{\omega M_{1}^{2} C_{D, \mathcal{R}}^{+}}{v_{d}|\Gamma|}}{1-\left(\frac{\omega}{\omega_{M}^{+}}\right)^{2}-\mathrm{i} \frac{\omega M_{1}^{2} C_{D, \mathcal{R}}^{+}}{v_{d}|\Gamma|}}\right),
$$

where $\omega_{M}^{+}:=\sqrt{\frac{\delta C_{D, \mathcal{R}}^{+}}{|D|}} v_{b}$ is the (periodic) Minnaert resonant frequency. Note that it is similar in expression to the usual Minnaert resonance $\omega_{M}$ [5, 27]. Actually, if the bubbles are small compared to the typical size of the lattice, and are far away from each other, then $\omega_{M}^{+} \approx \omega_{M}$. 
Remark 2.4. The term $\eta_{\mathrm{rad}}:=\frac{\omega M_{1}^{2} C_{D, \mathcal{R}}^{+}}{v_{d}|\Gamma|}$ in the denominator of (2.6) is called the radiative damping. It is possible to include more realistic damping effects [21]. In this case, one should replace $\eta_{\mathrm{rad}}$ by $\eta:=\eta_{\mathrm{rad}}+\eta_{\mathrm{other}}$, where $\eta_{\mathrm{other}}$ includes all the remaining sources of damping. Note that in the particular case where $\eta_{\mathrm{other}}=\eta_{\mathrm{rad}}$ so that $\eta=2 \eta_{\mathrm{rad}}$, then at the resonant frequency $\omega=\omega_{M}$ we obtain that $R(\omega) \approx 0$. This eventually explains the super-absorption phenomenon experimented in [24]: all the incoming energy is dissipated with damping effects.

The proof of Theorem 2.1 is given in the next section. It relies on the theory of periodic layer potentials.

\section{Proof of Theorem 2.1.}

\subsection{Periodic Green's functions}

We recall in this section the definition and properties of periodic layer potentials [6, Part 3]. Recall that $\mathcal{R}$ is a lattice of the plane $\mathbb{R}^{d-1}$. We let $\mathcal{R}^{*}$ be its reciprocal lattice, and denote by $\Gamma$ the unit cell of $\mathcal{R}$. For instance, if $d=2$ with $\mathcal{R}=a \mathbb{Z}$, then $\mathcal{R}^{*}=(2 \pi / a) \mathbb{Z}$ and $\Gamma=(-a / 2, a / 2)$.

Periodic Green's function without Dirichlet boundary condition. We first introduce, for $\mathbf{k} \in \mathbb{R}^{d}$, the periodic Green function $G_{\sharp}^{\mathbf{k}}$, solution to

$$
\left(\Delta+k^{2}\right) G_{\sharp}^{\mathbf{k}}(\mathbf{x}):=\sum_{\mathbf{R} \in \mathcal{R}} \mathrm{e}^{-\mathrm{i} \bar{k} \cdot \bar{x}} \delta_{\mathbf{R}}(\mathbf{x})=\sum_{\mathbf{R} \in \mathcal{R}} \mathrm{e}^{-\mathrm{i} \bar{k} \cdot \mathbf{R}} \delta_{\mathbf{R}}(\mathbf{x}),
$$

with the outgoing radiation condition. Here and after $\delta_{\mathbf{R}}(\mathbf{x})$ denotes the Dirac mass at the point $\mathbf{R}$. It holds that $G_{\sharp}^{\mathbf{k}}(\mathbf{x}, \mathbf{y})=G_{\sharp}^{k}(\mathbf{x}-\mathbf{y}, 0):=G_{\sharp}^{k}(\mathbf{x}-\mathbf{y})$.

The following lemma is needed.

Lemma 3.1. The solutions to $\left(\partial_{x x}^{2}+\alpha\right) f_{\alpha}=\delta_{0}$ are

$$
f_{\alpha}(x)=\left\{\begin{array}{l}
\frac{1}{2}|x|, \quad \alpha=0, \\
\frac{1}{2 \mathrm{i} \sqrt{\alpha}} \mathrm{e}^{\mathrm{i} \sqrt{\alpha}|x|}, \quad \alpha>0, \\
\frac{1}{2 \sqrt{-\alpha}} \mathrm{e}^{\sqrt{-\alpha}|x|}, \quad \alpha<0 .
\end{array}\right.
$$

Proof. It is enough to check that $f_{\alpha}$ is the solution to $\left(\partial_{x x}^{2}+\alpha\right) f_{\alpha}=\delta_{0}$. Recall that in the sense of distributions, $|x|^{\prime}=2 \Theta(x)-1$, where $\Theta$ is the Heaviside function $\Theta(x):=\mathbb{1}(x>0)$, and that $|x|^{\prime \prime}=2 \Theta^{\prime}(x)=2 \delta_{0}$. Note also that $\left(|x|^{\prime}\right)^{2}=1$. The proof follows by standard calculations.

The following result holds.

Lemma 3.2. If $\mathbf{k}=\mathbf{0}$, then

$$
G_{\sharp}^{\mathbf{0}}\left(\left(\bar{x}, x_{d}\right)\right)=\frac{\left|x_{d}\right|}{2|\Gamma|}-\sum_{\ell \in \mathcal{R}^{*} \backslash\{\mathbf{0}\}} \frac{1}{2|\Gamma||\ell|} \mathrm{e}^{-|\ell|\left|x_{d}\right|} \mathrm{e}^{\mathrm{i}(\ell \cdot \bar{x})} .
$$

If $\mathbf{k} \in \mathbb{R}^{d}$ satisfies $k^{2}<\inf \left\{|\ell-\bar{k}|, \ell \in \mathcal{R}^{*} \backslash\{\mathbf{0}\}\right\}$, then

$$
G_{\sharp}^{\mathbf{k}}\left(\left(\bar{x}, x_{d}\right)\right)=\frac{\mathrm{e}^{-\mathrm{i} \bar{k} \cdot \bar{x}} \mathrm{e}^{\mathrm{i} k_{d}\left|x_{d}\right|}}{2 \mathrm{i} k_{d}|\Gamma|}-\sum_{\ell \in \mathcal{R}^{*} \backslash\{0\}} \frac{\mathrm{e}^{-\mathrm{i} \bar{k} \cdot \bar{x}}}{2|\Gamma| \sqrt{|\ell-\bar{k}|^{2}-k^{2}}} \mathrm{e}^{-\sqrt{|\ell-\bar{k}|^{2}-k^{2}}\left|x_{d}\right|} \mathrm{e}^{\mathrm{i}(\ell \cdot \bar{x})} .
$$


Proof. In order to compute $G_{\sharp}^{\mathbf{k}}(\mathbf{x})$, we introduce $\widetilde{G_{\sharp}^{\mathbf{k}}}(\mathbf{x})=\mathrm{e}^{\mathrm{i} \hat{k} \cdot \overline{\mathbf{x}}} G_{\sharp}^{\mathbf{k}}(\mathbf{x})$, so that $G_{\sharp}^{\mathbf{k}}(\mathbf{x})=$ $\mathrm{e}^{-\mathrm{i} \bar{k} \cdot \overline{\mathbf{x}}} \widetilde{G_{\sharp}^{\mathbf{k}}}(\mathbf{x})$. In particular, $\widetilde{G_{\sharp}^{\mathbf{k}}}$ is the $\mathcal{R}$-periodic solution to $\left(\Delta-2 \mathrm{i} \bar{k} \cdot \nabla_{\bar{x}}+k_{d}^{2}\right) \widetilde{G_{\sharp}^{\mathbf{k}}}(\mathbf{x}):=$ $\sum_{\mathbf{R} \in \mathcal{R}} \delta_{\mathbf{R}}(\mathbf{x})$. We consider its Fourier expansion, and write

$$
\widetilde{G_{\sharp}^{\mathrm{k}}}\left(\bar{x}, x_{d}\right)=\sum_{\ell \in \mathcal{R}^{*}} c_{\ell}\left(x_{d}\right) \mathrm{e}^{\mathrm{i} \ell \cdot \bar{x}} .
$$

Thanks to the Poisson summation formula

$$
\sum_{\mathbf{R} \in \mathcal{R}} \delta(\mathbf{x}+\mathbf{R})=\frac{1}{|\Gamma|} \sum_{\ell \in \mathcal{R}^{*}} \mathrm{e}^{\mathrm{i} \ell \cdot \bar{x}}
$$

we obtain that $c_{\ell}$ must be the solution to

$$
\forall \ell \in \mathcal{R}^{*}, \quad\left(\partial_{x_{d} x_{d}}^{2}+k^{2}-|\ell-\bar{k}|^{2}\right) c_{\ell}=\frac{1}{|\Gamma|} \delta_{0} .
$$

The proof then follows from Lemma 3.1.

Remark 3.3. It is possible to simplify (3.2) following [16, pp. 813-814] (see for instance [4]).

From (3.3), we see that $G_{\sharp}^{\varepsilon \mathbf{k}}$ has a formal expansion of the form

$$
G_{\sharp}^{\varepsilon \mathbf{k}}(\mathbf{x})=\frac{1}{2 \mathrm{i} \varepsilon k_{d}|\Gamma|}+G_{0, \sharp}^{\mathbf{k}}(\mathbf{x})+\sum_{n=1}^{\infty} \varepsilon^{n} G_{n, \sharp}^{\mathbf{k}}(\mathbf{x}),
$$

where the operators $G_{n, \sharp}^{\mathbf{k}}$ can be computed explicitly. For instance, together with (3.2), we have

$$
G_{0, \sharp}^{\mathbf{k}}=\frac{k_{d}\left|x_{d}\right|-\bar{k} \cdot \bar{x}}{2 k_{d}|\Gamma|}-\sum_{\ell \in \mathcal{R}^{*} \backslash\{\mathbf{0}\}} \frac{\mathrm{e}^{-|\ell|\left|x_{d}\right|} \mathrm{e}^{\mathrm{i}(\ell \cdot \bar{x})}}{2|\Gamma||\ell|}=G_{\sharp}^{\mathbf{0}}-\frac{\bar{k} \cdot \bar{x}}{2 k_{d}|\Gamma|} .
$$

We will also need the exact formula for $G_{1, \sharp}^{\mathbf{k}}$. After some straightforward calculations we find that

$$
G_{1, \sharp}^{\mathbf{k}}(\mathbf{x})=\frac{\mathrm{i}\left(k_{d}\left|x_{d}\right|-\bar{k} \cdot \bar{x}\right)^{2}}{4 k_{d}|\Gamma|}-\bar{k} \cdot g_{1, \sharp}(\mathbf{x}),
$$

where $g_{1, \sharp}$ is a function independent of $\mathbf{k}$. Explicitly,

$$
g_{1, \sharp}(\mathbf{x}):=\sum_{\ell \in \mathcal{R}^{*} \backslash\{\mathbf{0}\}} \frac{\mathrm{e}^{-|\ell|\left|x_{d}\right|} \mathrm{e}^{\mathrm{i}(\ell \cdot \bar{x})}}{2|\Gamma||\ell|}\left(\frac{\ell}{|\ell|^{2}}+\frac{\ell}{|\ell|}\left|x_{d}\right|-\mathrm{i} \bar{x}\right) .
$$

By change of variable $\ell \rightarrow-\ell$, we obtain that

$$
g_{1, \sharp}(\mathbf{x})=\frac{\mathrm{i}}{2} \sum_{\ell \in \mathcal{R}^{*} \backslash\{\mathbf{0}\}} \frac{\mathrm{e}^{-|\ell|\left|x_{d}\right|}}{|\Gamma||\ell|}\left(\left(\frac{\ell}{|\ell|^{2}}+\frac{\ell}{|\ell|}\left|x_{d}\right|\right) \sin (\ell \cdot \bar{x})-\bar{x} \cos (\ell \cdot \bar{x})\right) .
$$

In particular, we see that $g_{1, \sharp}$ satisfies the symmetry relations

$$
g_{1, \sharp}\left(\bar{x}, x_{d}\right)=g_{1, \sharp}\left(\bar{x},-x_{d}\right)=-g_{1, \sharp}\left(-\bar{x}, x_{d}\right) .
$$


Note that there is a singularity in (3.4) as $\varepsilon$ goes to 0. Finally, expanding (3.1) in powers of $\varepsilon$ leads to the equations

$$
\left\{\begin{aligned}
& \Delta G_{0, \sharp}^{\mathbf{k}}=\sum_{\mathbf{R} \in \mathcal{R}} \delta_{\mathbf{R}}, \\
& \Delta G_{1, \sharp}^{\mathbf{k}}=\sum_{\mathbf{R} \in \mathcal{R}}-\mathrm{i} \mathbf{R} \delta_{\mathbf{R}}, \\
& \Delta G_{n+2, \sharp}^{\mathbf{k}}+k^{2} G_{n, \sharp}^{\mathbf{k}}=\sum_{\mathbf{R} \in \mathcal{R}} \frac{(-\mathrm{i} \bar{k} \cdot \mathbf{R})^{n+2}}{(n+2) !} \delta_{\mathbf{R}}, \quad \forall n \geq 2 .
\end{aligned}\right.
$$

The periodic Green function would be an adequate tool to study the problem without the Dirichlet boundary condition $U^{\varepsilon}=0$ on $\partial \mathbb{R}_{+}^{d}$. In this article however, we study the problem with the Dirichlet boundary condition to explain the phenomenon seen in [24] for instance.

Periodic Green's function with Dirichlet boundary condition We introduce the Dirichlet Green function defined by

$$
G_{+}^{\mathbf{k}}(\mathbf{x}, \mathbf{y}):=G_{\sharp}^{\mathbf{k}}\left(\left(\bar{x}-\bar{y}, x_{d}-y_{d}\right)\right)-G_{\sharp}^{\mathbf{k}}\left(\left(\bar{x}-\bar{y}, x_{d}+y_{d}\right)\right) .
$$

This Green function is no longer translational invariant (in the sense $G_{+}^{\mathbf{k}}(\mathbf{x}, \mathbf{y}) \neq G_{+}^{\mathbf{k}}(\mathbf{x}-\mathbf{y}, \mathbf{0})$ ), but satisfies the Dirichlet boundary condition $G_{+}^{\mathbf{k}}=0$ on $\partial \mathbb{R}_{+}^{d}$. From (3.3), we deduce that $G_{+}^{\varepsilon \mathbf{k}}$ admits an expansion of the form

$$
G_{+}^{\varepsilon \mathbf{k}}(\mathbf{x}, \mathbf{y})=\sum_{n=0}^{\infty} \varepsilon^{n} G_{n,+}^{\mathbf{k}}(\mathbf{x}, \mathbf{y})
$$

where

$$
G_{n,+}^{\mathbf{k}}(\mathbf{x}, \mathbf{y}):=G_{n, \sharp}^{\mathbf{k}}\left(\left(\bar{x}-\bar{y}, x_{d}-y_{d}\right)\right)-G_{n, \sharp}^{\mathbf{k}}\left(\left(\bar{x}-\bar{y}, x_{d}+y_{d}\right)\right) .
$$

Note that $G_{+}^{\varepsilon \mathbf{k}}$ is no longer singularity as $\varepsilon$ goes to 0 . This makes the problem with Dirichlet boundary condition easier to study analytically. Moreover, from (3.5) we can check that

$$
G_{\mathbf{0},+}^{\mathbf{k}}(\mathbf{x}, \mathbf{y})=G_{+}^{\mathbf{k}=\mathbf{0}}(\mathbf{x}, \mathbf{y}) .
$$

Note that this equality does not hold for the periodic Green function without Dirichlet boundary condition (see (3.5). We also need the expression of $G_{1,+}^{\mathrm{k}}$. We get

$$
G_{1,+}^{\mathbf{k}}(\mathbf{x}, \mathbf{y})=\frac{-\mathrm{i}}{2|\Gamma|}\left(2 k_{d} x_{d} y_{d}+\bar{k} \cdot(\bar{x}-\bar{y})\left(\left|x_{d}-y_{d}\right|-\left|x_{d}+y_{d}\right|\right)\right)-\bar{k} \cdot g_{1,+}(\mathbf{x}, \mathbf{y})
$$

with

$$
g_{1,+}:=g_{1, \sharp}\left(\bar{x}-\bar{y}, x_{d}-y_{d}\right)-g_{1, \sharp}\left(\bar{x}-\bar{y}, x_{d}+y_{d}\right) .
$$

From (3.8), we see that $g_{1,+}$ satisfies the symmetry relation

$$
g_{1,+}(\mathbf{x}, \mathbf{y})=-g_{1,+}(\mathbf{y}, \mathbf{x}) .
$$

Finally, from (3.9), we deduce that

$$
\Delta_{\mathbf{y}} G_{1,+}^{\mathbf{k}}(\mathbf{x}, \mathbf{y})=\sum_{\mathbf{R} \in \mathcal{R}}(-\mathrm{i} \bar{k} \cdot \mathbf{R}) \delta_{\mathbf{R}}(\bar{x}-\bar{y})\left(\delta_{0}\left(x_{d}-y_{d}\right)-\delta_{0}\left(x_{d}+y_{d}\right)\right),
$$


and that, for $n \in \mathbb{N}$,

$$
\Delta_{\mathbf{y}} G_{n+2,+}^{\mathbf{k}}(\mathbf{x}, \mathbf{y})+k^{2} G_{n,+}^{\mathbf{k}}(\mathbf{x}, \mathbf{y})=\sum_{\mathbf{R} \in \mathcal{R}} \frac{(-\mathrm{i} \bar{k} \cdot \mathbf{R})^{n+2}}{(n+2) !} \delta_{\mathbf{R}}(\bar{x}-\bar{y})\left(\delta_{0}\left(x_{d}-y_{d}\right)-\delta_{0}\left(x_{d}+y_{d}\right)\right) .
$$

In particular, if $\mathbf{x}, \mathbf{y} \in \bar{D}$, then, since $\delta_{\mathbf{R}}(\bar{x}-\bar{y})=0$ except for $\mathbf{R}=\mathbf{0}$ (and $\bar{x}=\bar{y}$ ), we have

$$
\Delta_{\mathbf{x}} G_{1,+}^{\mathbf{k}}=\Delta_{\mathbf{y}} G_{1,+}^{\mathbf{k}}=0 \quad \text { and } \quad \forall n \in \mathbb{N}, \quad \Delta_{\mathbf{y}} G_{n+2,+}^{\mathbf{k}}(\mathbf{x}, \mathbf{y})=-k^{2} G_{n,+}^{\mathbf{k}}(\mathbf{x}, \mathbf{y})
$$

\subsection{Periodic-Dirichlet layer potentials}

We now introduce the periodic-Dirichlet layer potential operators. We denote by $H^{-1 / 2}:=$ $H^{-1 / 2}(\partial D)$ and by $H^{1 / 2}:=H^{1 / 2}(\partial D)$ the usual fractional Sobolev spaces on surfaces [26]. In the sequel, we use $\langle\cdot, \cdot\rangle$ for the $H^{-1 / 2}, H^{1 / 2}$ duality pairing. We also introduce $H_{0}^{-1 / 2}:=$ $\left\{f \in H^{-1 / 2},\langle f, 1\rangle=0\right\}$. The periodic-Dirichlet single-layer potential $\mathcal{S}_{+}^{\mathbf{k}}: H^{-1 / 2} \rightarrow H^{1 / 2}$ and the periodic-Dirichlet Dirichlet-to-Neumann operator $\mathcal{K}_{+}^{\mathbf{k}, *}: H^{-1 / 2} \rightarrow H^{-1 / 2}$ are respectively defined, for smooth functions $\psi \in C^{\infty}(\partial D)$ by

$\forall \mathbf{x} \in \partial D, \quad \mathcal{S}_{+}^{\mathbf{k}}[\psi](\mathbf{x}):=\int_{\partial D} G_{+}^{\mathbf{k}}(\mathbf{x}, \mathbf{y}) \psi(\mathbf{y}) \mathrm{d} \sigma(\mathbf{y}), \quad \mathcal{K}_{+}^{\mathbf{k}, *}[\psi](\mathbf{x}):=\int_{\partial D} \frac{\partial G_{+}^{\mathbf{k}}}{\partial \nu_{\mathbf{x}}}(\mathbf{x}, \mathbf{y}) \psi(\mathbf{y}) \mathrm{d} \sigma(\mathbf{y})$.

For simplicity, we write $\mathcal{K}_{+}^{*}:=\mathcal{K}_{+}^{\mathbf{0}, *}$ and $\mathcal{S}_{+}:=\mathcal{S}_{+}^{\mathbf{0}}$. We also introduce the operator $\widetilde{\mathcal{S}_{+}^{\mathrm{k}}}: H^{-1 / 2} \rightarrow$ $H_{\text {loc }}^{1}\left(\mathbb{R}^{d}\right)$ defined by

$$
\forall \psi \in H^{-1 / 2}, \quad \forall \mathbf{x} \in \mathbb{R}^{d}, \quad \widetilde{\mathcal{S}_{+}^{\mathbf{k}}}[\psi](\mathbf{x}):=\int_{\partial D} G_{+}^{\mathbf{k}}(\mathbf{x}, \mathbf{y}) \psi(\mathbf{y}) \mathrm{d} \sigma(\mathbf{y}) .
$$

We have the following standard result.

\section{Lemma 3.4.}

(i) For all $\mathbf{k} \in \mathbb{R}^{d}$, the operator $\mathcal{S}_{+}^{\mathbf{k}}: H^{-1 / 2} \rightarrow H^{1 / 2}$ is an bounded operator with bounded inverse. Moreover, it holds that $\mathcal{S}_{+}^{*}=\mathcal{S}_{+}$.

(ii) For all $\mathbf{k} \in \mathbb{R}^{d}$, the operator $\mathcal{K}_{+}^{\mathbf{k}, *}$ is a compact operator on $H^{-1 / 2}$, and the operator $\mathcal{K}_{+}^{\mathbf{k}}$ is a compact operator on $H^{1 / 2}$.

(iii) (jump formulae) It holds that

$$
\left.\widetilde{\mathcal{S}_{+}^{\mathbf{k}}}\right|_{+}=\left.\widetilde{\mathcal{S}_{+}^{\mathbf{k}}}\right|_{-}=\mathcal{S}_{+}^{\mathbf{k}} \quad \text { and }\left.\quad\left(\partial_{\nu} \widetilde{\mathcal{S}_{+}^{\mathbf{k}}}\right)\right|_{ \pm}= \pm \frac{1}{2}+\mathcal{K}_{+}^{\mathbf{k}, *}
$$

(iv) It holds that $\sigma\left(\mathcal{K}_{+}^{*}\right)=\sigma\left(\mathcal{K}_{+}\right) \subset(-1 / 2,1 / 2]$ and that

$$
\operatorname{Ker}\left(\mathcal{K}_{+}-\frac{1}{2}\right)=\operatorname{Span}\left\{\mathbb{1}_{\partial D}\right\}, \quad \text { and } \operatorname{Ker}\left(\mathcal{K}_{+}^{*}-\frac{1}{2}\right)=\operatorname{Span}\left\{\psi_{0}^{+}\right\}
$$

where $\mathbb{1}_{\partial D} \in H^{1 / 2}$ is the constant function with value 1 on $\partial D$, and where $\psi_{0}^{+} \in H^{-1 / 2}$ is such that $\left\langle\mathbb{1}_{\partial D}, \psi_{0}^{+}\right\rangle=1$.

(v) The operator $\left(-\frac{1}{2}+\mathcal{K}_{+}^{*}\right)$ acting on $H_{0}^{-1 / 2}$ is invertible with bounded inverse. 
We now introduce two constants. Recall that $\psi_{0}^{+}$is defined by $\operatorname{Ker}\left(\mathcal{K}_{+}^{*}-\frac{1}{2}\right)=\operatorname{Span}\left\{\psi_{0}^{+}\right\}$ and $\left\langle\mathbb{1}_{\partial D}, \psi_{0}^{+}\right\rangle=1$. We define

$$
\begin{aligned}
C_{D, \mathcal{R}}^{+} & =-\left\langle\mathcal{S}_{+}^{-1} \mathbb{1}_{\partial D}, \mathbb{1}_{\partial D}\right\rangle \\
M_{1} & =\left\langle\psi_{0}^{+},\left.x_{d}\right|_{\partial D}\right\rangle .
\end{aligned}
$$

We have the following result.

Lemma 3.5. (i) For all $\mathbf{x} \in D, \widetilde{\mathcal{S}_{+}}\left[\psi_{0}^{+}\right](\mathbf{x})=-1 / C_{D, \mathcal{R}}^{+}$. Especially, $\mathcal{S}_{+}\left[\psi_{0}^{+}\right]=-\left(1 / C_{D, \mathcal{R}}^{+}\right) \mathbb{1}_{\partial D}$.

(ii) Let $\psi_{1}^{+}:=\left(-\frac{1}{2}+\mathcal{K}_{+}^{*}\right)^{-1}\left[\nu_{d}\right] \in H_{0}^{-1 / 2}$, where $\nu_{d}$ is the d-component of the outward normal $\nu$ to $\partial D$. Then it holds that

$$
\forall \mathbf{x} \in \partial D, \quad \mathcal{S}_{+}\left[\psi_{1}^{+}\right](\mathbf{x})=\left.x_{d}\right|_{\partial D}-M_{1} \quad \text { and } \quad \forall \mathbf{x} \in D, \quad \widetilde{\mathcal{S}_{+}}\left[\psi_{1}^{+}\right](\mathbf{x})=x_{d}-M_{1},
$$

In particular, $\mathcal{S}_{+}^{-1}\left[\left.x_{d}\right|_{\partial D}\right]=\psi_{1}^{+}-C_{D, \mathcal{R}}^{+} M_{1} \psi_{0}^{+}$.

Proof. The proof of (i) is straightforward. Let us prove (ii). For all $M \in \mathbb{R}$, the function $f(\mathbf{x}):=x_{d}-M$ satisfies $\Delta f=0$ and $\left.\partial_{\nu} f\right|_{-}=\nu_{d}$. Together with the jump formulae, we deduce that

$$
\forall \mathbf{x} \in D, \quad \widetilde{\mathcal{S}_{+}}\left[\psi_{1}^{+}\right](\mathbf{x})=x_{d}-M,
$$

where $M$ is chosen so that $\psi_{1}^{+} \in L_{0}^{2}$. To calculate $M$, we notice that

$$
0=\left\langle\mathbb{1}_{\partial D}, \psi_{1}^{+}\right\rangle=\left\langle\mathbb{1}_{\partial D}, \mathcal{S}_{+}^{-1}\left[x_{d}-M \mathbb{1}_{\partial D}\right]\right\rangle=\left\langle\mathcal{S}_{+}^{-1}[\mathbb{1}], x_{d}-M \mathbb{1}_{\partial D}\right\rangle=-C_{D, \mathcal{R}}^{+}\left\langle\psi_{0}^{+}, x_{d}-M \mathbb{1}_{\partial D}\right\rangle .
$$

The result follows.

Definition 3.6. We call the constant $C_{D, \mathcal{R}}^{+}$the periodic capacity of $D$ with respect to the lattice $\mathcal{R}$.

Remark 3.7. The periodic capacity $C_{D, \mathcal{R}}^{+}$is positive. Both $C_{D, \mathcal{R}}^{+}$and $M_{1}$ depend on the lattice $\mathcal{R}$.

\subsection{Equivalent formulation}

We now rescale the problem (2.1). Recall that $\delta=\delta_{\varepsilon}=\mu \varepsilon^{2}$. In the sequel, we denote by $\mathbf{X}$ the macroscopic variable and by $\mathbf{x}:=\frac{\mathbf{x}}{\varepsilon}$ the microscopic one. We denote by $u(\mathbf{x}):=U(\varepsilon \mathbf{x})$. With this change of variable, (2.1) is equivalent to

$$
\left\{\begin{array}{l}
\left(\Delta+\varepsilon^{2} k^{2}\right) u^{\varepsilon}=0 \text { on } \mathbb{R}^{d}-\backslash \overline{\Omega^{1}} \\
\left(\Delta+\varepsilon^{2} k_{b}^{2}\right) u^{\varepsilon}=0 \text { on } \Omega^{1}, \\
u^{\varepsilon}\left|+=u^{\varepsilon}\right|_{-} \text {on } \partial \Omega^{1}, \\
\left.\partial_{\nu} u^{\varepsilon}\right|_{-}=\left.\mu \varepsilon^{2} \partial_{\nu} u^{\varepsilon}\right|_{+} \text {on } \partial \Omega^{1}, \\
u^{\varepsilon}-U_{\varepsilon \mathbf{k}}^{\text {in }} \text { satisfies the outgoing radiation condition, } \\
u^{\varepsilon}=0 \text { on } \partial \mathbb{R}_{+}^{2} .
\end{array}\right.
$$

We use layer potentials to solve (3.18). We consider the case when the incidence angle is such that $\bar{k}^{2} \leq k_{b}^{2}$. The case $\bar{k}^{2} \geq k_{b}^{2}$ can be treated in a similar manner. We set $k_{b, d}=\sqrt{k_{b}^{2}-\bar{k}^{2}}$, 
and denote by $\mathbf{k}_{b}=\left(\bar{k}, k_{b, d}\right) \in \mathbb{R}^{d}$ the vector such that $\left|\mathbf{k}_{b}\right|=k_{b}$. The solution to (3.18) can be represented by

$$
u^{\varepsilon}(\mathbf{x})=\left\{\begin{array}{l}
U_{\varepsilon \mathbf{k}}^{\text {in }}\left(\bar{x}, x_{d}\right)-U_{\varepsilon \mathbf{k}}^{\text {in }}\left(\bar{x},-x_{d}\right)+\widetilde{\mathcal{S}_{+}^{\varepsilon \mathbf{k}}}[\psi](\mathbf{x}) \text { for } \quad \mathbf{x} \in \mathbb{R}^{d} \backslash \overline{\Omega^{1}}, \\
\widetilde{\mathcal{S}_{+}^{\varepsilon \mathbf{k}_{b}}}\left[\psi_{b}\right](\mathbf{x}) \text { for } \quad \mathbf{x} \in \Omega^{1},
\end{array}\right.
$$

where $\psi_{b}, \psi \in H^{-1 / 2}$ are surface potentials. In the sequel, we denote by $\mathcal{H}^{--}:=H^{-1 / 2} \times H^{-1 / 2}$ and by $\mathcal{H}^{+-}:=H^{1 / 2} \times H^{-1 / 2}$. After some straightforward calculations and using Lemma 3.4, we see that (3.18) is equivalent to finding $\Psi(\varepsilon)=\left(\psi_{b}, \psi\right)^{t} \in \mathcal{H}^{--}$such that

$$
\mathcal{A}(\varepsilon) \Psi(\varepsilon)=F(\varepsilon),
$$

where

$$
\mathcal{A}(\varepsilon):=\left(\begin{array}{cc}
\mathcal{S}_{+}^{\varepsilon \mathbf{k}_{b}} & -\mathcal{S}_{+}^{\varepsilon \mathbf{k}} \\
-\frac{1}{2}+\mathcal{K}_{+}^{\varepsilon \mathbf{k}_{b}, *} & -\mu \varepsilon^{2}\left(\frac{1}{2}+\mathcal{K}_{+}^{\varepsilon \mathbf{k}, *}\right)
\end{array}\right)
$$

and

$$
\begin{aligned}
F(\varepsilon) & :=\left(\begin{array}{c}
\left.U_{\varepsilon \mathbf{k}}^{\mathrm{in}}\left(\bar{x}, x_{d}\right)\right|_{+}-\left.U_{\varepsilon \mathbf{k}}^{\mathrm{in}}\left(\bar{x},-x_{d}\right)\right|_{+} \\
\left.\delta_{\varepsilon} \partial_{\nu}\left(U_{\varepsilon \mathbf{k}}^{\mathrm{in}}\left(\bar{x}, x_{d}\right)-U_{\varepsilon \mathbf{k}}^{\mathrm{in}}\left(\bar{x},-x_{d}\right)\right)\right|_{+}
\end{array}\right) \\
& =-2 \mathrm{i} u_{0}\left(\begin{array}{c}
\sin \left(\varepsilon k_{d} x_{d}\right) \\
\varepsilon^{3} \mu\left(k_{d} \nu_{d} \cos \left(\varepsilon k_{d} x_{d}\right)-\mathrm{i} \bar{k} \cdot \bar{\nu} \sin \left(\varepsilon k_{d} \nu_{d}\right)\right)
\end{array}\right) \mathrm{e}^{-\mathrm{i} \varepsilon \bar{k} \cdot \bar{x}} .
\end{aligned}
$$

By Lemma 3.4, $\mathcal{A}(\varepsilon)$ is a bounded operator from $\mathcal{H}^{--}$to $\mathcal{H}^{+-}$. We study (3.20) using Taylor expansion. Following the decomposition (3.11), we write

$$
\mathcal{S}_{+}^{\varepsilon \mathbf{k}}=\mathcal{S}_{+}+\sum_{n=1}^{\infty} \varepsilon^{n} \mathcal{S}_{n,+}^{\mathbf{k}} \quad \text { and } \quad \mathcal{K}_{+}^{\varepsilon \mathbf{k}, *}=\mathcal{K}_{+}^{*}+\sum_{n=1}^{\infty} \varepsilon^{n} \mathcal{K}_{n,+}^{\mathbf{k}, *},
$$

where the convergence holds in $\mathcal{B}\left(H^{-1 / 2}, H^{1 / 2}\right)$ and $\mathcal{B}\left(H^{-1 / 2}\right)$ respectively, and where, for $n \in \mathbb{N}^{*}, \psi \in H^{-1 / 2}$ and $\mathbf{x} \in \partial D$,

$$
\mathcal{S}_{n,+}^{\mathbf{k}}[\psi](\mathbf{x}):=\int_{\partial D} G_{n,+}^{\mathbf{k}}(\mathbf{x}, \mathbf{y}) \psi(\mathbf{y}) \mathrm{d} \sigma(\mathbf{y}) \quad \text { and } \quad \mathcal{K}_{n,+}^{*}[\psi](\mathbf{x}):=\int_{\partial D} \frac{\partial G_{n,+}^{\mathbf{k}}}{\partial \nu_{\mathbf{x}}}(\mathbf{x}, \mathbf{y}) \psi(\mathbf{y}) \mathrm{d} \sigma(\mathbf{y}) .
$$

Here, $\mathcal{B}\left(H^{-1 / 2}, H^{1 / 2}\right)$ denotes the set of linear bounded operators from $H^{-1 / 2}$ onto $H^{1 / 2}$ and $\mathcal{B}\left(H^{-1 / 2}\right)$ is the set of linear bounded operators on $H^{-1 / 2}$. Then we write

$$
\mathcal{A}(\varepsilon)=\mathcal{A}_{0}+\mathcal{B}(\varepsilon) \quad \text { with } \quad \mathcal{B}(\varepsilon):=\sum_{n=1}^{\infty} \varepsilon^{n} \mathcal{A}_{n},
$$

where

$$
\mathcal{A}_{0}:=\left(\begin{array}{cc}
\mathcal{S}_{+} & -\mathcal{S}_{+} \\
-\frac{1}{2}+\mathcal{K}_{+}^{*} & 0
\end{array}\right), \quad \mathcal{A}_{1}:=\left(\begin{array}{cc}
\mathcal{S}_{1,+}^{\mathbf{k}_{b}} & -\mathcal{S}_{1,+}^{\mathbf{k}} \\
\mathcal{K}_{1,+}^{\mathbf{k}_{b}, *} & 0
\end{array}\right), \quad \mathcal{A}_{2}:=\left(\begin{array}{cc}
\mathcal{S}_{2,+}^{\mathbf{k}_{b}} & -\mathcal{S}_{2,+}^{\mathbf{k}} \\
\mathcal{K}_{2,+}^{\mathbf{k}_{b}, *} & -\mu\left(\frac{1}{2}+\mathcal{K}_{+}^{*}\right.
\end{array}\right),
$$

and, for $n \geq 3$,

$$
\mathcal{A}_{n}:=\left(\begin{array}{cc}
\mathcal{S}_{n,+}^{\mathbf{k}_{b}} & -\mathcal{S}_{n,+}^{\mathbf{k}} \\
\mathcal{K}_{n,+}^{\mathbf{k}_{b, *}} & -\mu \mathcal{K}_{n-2,+}^{\mathbf{k}, *}
\end{array}\right)
$$


It is standard to check that the convergence holds in $\mathcal{B}\left(\mathcal{H}^{--}, \mathcal{H}^{+-}\right)$. We would like to approximate $\mathcal{A}(\varepsilon)^{-1}$ by $\mathcal{A}_{0}^{-1}$. Unfortunately, this is not possible, since the operator $\mathcal{A}_{0}$ is not invertible. It is indeed easy to check that $\operatorname{Ker}\left(\mathcal{A}_{0}\right)=\operatorname{Span}\left\{\left(\begin{array}{c}\psi_{0}^{+} \\ \psi_{0}^{+}\end{array}\right)\right\}$. In order to handle this difficulty, we perturb the operator $\mathcal{A}_{0}$ (see also the method used in [5]). We introduce a rank-1 projection operator $\Pi \in \mathcal{B}\left(H^{-1 / 2}\right)$ defined by

$$
\forall \psi \in H^{-1 / 2}, \quad \Pi[\psi]=\left\langle\mathbb{1}_{\partial D}, \psi\right\rangle \psi_{0}^{+},
$$

and we set

$$
\widetilde{\mathcal{A}_{0}}:=\mathcal{A}_{0}+\mathcal{P} \quad \text { and } \quad \widetilde{\mathcal{A}}(\varepsilon):=\mathcal{A}(\varepsilon)+\mathcal{P}, \quad \text { with } \quad \mathcal{P}:=\left(\begin{array}{cc}
0 & 0 \\
\Pi & 0
\end{array}\right)
$$

Lemma 3.8. The operator $\widetilde{\mathcal{A}_{0}}$ is bounded and is invertible with inverse

$$
{\widetilde{\mathcal{A}_{0}}}^{-1}=\left(\begin{array}{cc}
0 & \left(-\frac{1}{2}+\mathcal{K}_{+}^{*}+\Pi\right)^{-1} \\
-\mathcal{S}_{+}^{-1} & \left(-\frac{1}{2}+\mathcal{K}_{+}^{*}+\Pi\right)^{-1}
\end{array}\right) .
$$

Recall that we want to calculate $\Psi$, the solution to (3.20). We introduce

$$
Q:=\left(\begin{array}{cc}
\Pi & 0 \\
0 & 0
\end{array}\right), \quad \Psi_{-}:=(1-Q) \Psi \quad \text { and } \quad \Psi_{+}:=Q \Psi \quad \text { so that } \quad \Psi=\Psi_{-}+\Psi_{+} .
$$

For the sake of clarity, we introduce the vectors $\Phi_{1}, \Phi_{2} \in \mathcal{H}^{--}$and $\Phi_{3}, \Phi_{4} \in \mathcal{H}^{+-}$defined by

$$
\Phi_{1}:=\left(\begin{array}{c}
\psi_{0}^{+} \\
0
\end{array}\right), \quad \Phi_{2}:=\left(\begin{array}{c}
0 \\
\psi_{0}^{+}
\end{array}\right), \quad \Phi_{3}:=\left(\begin{array}{c}
\mathbb{1}_{\partial D} \\
0
\end{array}\right), \quad \text { and } \quad \Phi_{4}:=\left(\begin{array}{c}
0 \\
\mathbb{1}_{\partial D}
\end{array}\right) .
$$

Note that $\Psi_{+}=\alpha \Phi_{1}$ for some $\alpha \in \mathbb{C}$. The equation (3.20) is therefore equivalent to

$$
\left(\widetilde{\mathcal{A}_{0}}+\mathcal{B}(\varepsilon)-\mathcal{P}\right)\left(\alpha \Phi_{1}+\Psi_{-}\right)=F(\varepsilon) .
$$

From Lemma 3.8, we deduce that for $\varepsilon$ small enough, the operator $\widetilde{\mathcal{A}_{0}}+\mathcal{B}(\varepsilon)$ is invertible, and that its inverse is given by the Neumann series

$$
\widetilde{\mathcal{A}}(\varepsilon)^{-1}=\left(\widetilde{\mathcal{A}_{0}}+\mathcal{B}(\varepsilon)\right)^{-1}={\widetilde{\mathcal{A}_{0}}}^{-1}+\sum_{n=1}^{\infty}(-1)^{n}\left({\widetilde{\mathcal{A}_{0}}}^{-1} \mathcal{B}(\varepsilon)\right)^{n}{\widetilde{\mathcal{A}_{0}}}^{-1}
$$

where the convergence holds in $\mathcal{B}\left(\mathcal{H}^{+-}, \mathcal{H}^{--}\right)$. Applying $\left(\widetilde{\mathcal{A}_{0}}+\mathcal{B}(\varepsilon)\right)^{-1}$ to both sides of $(3.24)$, and using the fact that $\mathcal{P} \Phi_{1}=\Phi_{2}$ and that $\mathcal{P} \Psi_{-}=0$, we obtain

$$
\alpha \Phi_{1}+\Psi_{-}-\alpha\left(\widetilde{\mathcal{A}_{0}}+\mathcal{B}(\varepsilon)\right)^{-1} \Phi_{2}=\left(\widetilde{\mathcal{A}_{0}}+\mathcal{B}(\varepsilon)\right)^{-1} F(\varepsilon) .
$$

Finally, we notice that $\left\langle\Phi_{3}, \Psi_{-}\right\rangle=0$, so that, by taking the duality product with $\Phi_{3}$, we obtain that (3.20) is equivalent to

$$
\left\{\begin{array}{l}
\alpha=\frac{\left\langle\Phi_{3},\left(\widetilde{\mathcal{A}_{0}}+\mathcal{B}(\varepsilon)\right)^{-1} F(\varepsilon)\right\rangle}{1-\left\langle\Phi_{3},\left(\widetilde{\mathcal{A}_{0}}+\mathcal{B}(\varepsilon)\right)^{-1} \Phi_{2}\right\rangle}, \\
\Psi_{-}=\left(\widetilde{\mathcal{A}_{0}}+\mathcal{B}(\varepsilon)\right)^{-1} F(\varepsilon)-\alpha \Phi_{1}+\alpha\left(\widetilde{\mathcal{A}_{0}}+\mathcal{B}(\varepsilon)\right)^{-1} \Phi_{2} .
\end{array}\right.
$$




\subsection{Asymptotic expansions}

We now solve (3.26) using asymptotic expansions in $\varepsilon$. We first need some estimates.

Lemma 3.9. The following identities hold.

$$
\begin{aligned}
& \text { (i) } \mathcal{K}_{1,+}^{\mathbf{k}}\left[\mathbb{1}_{\partial D}\right]=0, \\
& \text { (ii) } \mathcal{K}_{2,+}^{\mathbf{k}}\left[\mathbb{1}_{\partial D}\right](\mathbf{x})=-k^{2} \int_{D} G_{+}^{\mathbf{0}}(\mathbf{x}, \mathbf{y}) \mathrm{d} \mathbf{y} \\
& \text { (iii) } \mathcal{K}_{3,+}^{\mathbf{k}}\left[\mathbb{1}_{\partial D}\right](\mathbf{x})=-k^{2} \int_{D} G_{1,+}^{\mathbf{k}}(\mathbf{x}, \mathbf{y}) \mathrm{d} \mathbf{y}
\end{aligned}
$$

Proof. First, we recall that $G_{1,+}^{\mathbf{k}}(\mathbf{x}, \mathbf{y})$ satisfies (3.14). In particular,

$$
\text { (i) } \quad \mathcal{K}_{1,+}^{\mathbf{k}}\left[\mathbb{1}_{\partial D}\right](\mathbf{x})=\int_{\partial D} \partial_{\nu_{\mathbf{y}}} G_{1,+}^{\mathbf{k}}(\mathbf{x}, \mathbf{y}) \mathrm{d} \sigma(\mathbf{y})=\int_{D} \Delta_{\mathbf{x}} G_{1,+}^{\mathbf{k}}(\mathbf{x}, \mathbf{y}) \mathrm{d} \mathbf{x}=0
$$

Also, using (3.14), we obtain

(ii) $\mathcal{K}_{2,+}^{\mathbf{k}}\left[\mathbb{1}_{\partial D}\right](\mathbf{x})=\int_{\partial D} \partial_{\nu_{\mathbf{y}}} G_{2,+}^{\mathbf{k}}(\mathbf{x}, \mathbf{y}) \mathrm{d} \sigma(\mathbf{y})=\int_{D} \Delta_{\mathbf{y}} G_{2,+}^{\mathbf{k}}(\mathbf{x}, \mathbf{y}) \mathrm{d} \mathbf{y}=-k^{2} \int_{D} G_{+}^{\mathbf{0}}(\mathbf{x}, \mathbf{y}) \mathrm{d} \mathbf{y}$, and similarly,

$$
\text { (iii) } \mathcal{K}_{3,+}^{\mathbf{k}}\left[\mathbb{1}_{\partial D}\right](\mathbf{x})=\int_{\partial D} \partial_{\nu_{\mathbf{y}}} G_{3,+}^{\mathbf{k}}(\mathbf{x}, \mathbf{y}) \mathrm{d} \sigma(\mathbf{y})=-k^{2} \int_{D} G_{1,+}^{\mathbf{k}}(\mathbf{x}, \mathbf{y}) \mathrm{d} \mathbf{y} .
$$

We start with the calculation of $\alpha$.

- Evaluation of $\left\langle\Phi_{3},\left(\widetilde{\mathcal{A}_{0}}+\mathcal{B}(\varepsilon)\right)^{-1} \Phi_{2}\right\rangle$. From (3.25), we have

$$
\begin{aligned}
\left(\widetilde{\mathcal{A}_{0}}+\mathcal{B}(\varepsilon)\right)^{-1} & ={\widetilde{\mathcal{A}_{0}}}^{-1}-\varepsilon\left({\widetilde{\mathcal{A}_{0}}}^{-1} \mathcal{A}_{1}{\widetilde{\mathcal{A}_{0}}}^{-1}\right)+\varepsilon^{2}\left(\left[{\widetilde{\mathcal{A}_{0}}}^{-1} \mathcal{A}_{1}\right]^{2}{\widetilde{\mathcal{A}_{0}}}^{-1}-{\widetilde{\mathcal{A}_{0}}}^{-1} \mathcal{A}_{2}{\widetilde{\mathcal{A}_{0}}}^{-1}\right) \\
& -\varepsilon^{3}\left(\left[{\widetilde{\mathcal{A}_{0}}}^{-1} \mathcal{A}_{1}\right]^{3}{\widetilde{\mathcal{A}_{0}}}^{-1}+{\widetilde{\mathcal{A}_{0}}}^{-1} \mathcal{A}_{3}{\widetilde{\mathcal{A}_{0}}}^{-1}\right) \\
& +\varepsilon^{3}\left({\widetilde{\mathcal{A}_{0}}}^{-1} \mathcal{A}_{1}{\widetilde{\mathcal{A}_{0}}}^{-1} \mathcal{A}_{2}{\widetilde{\mathcal{A}_{0}}}^{-1}+{\widetilde{\mathcal{A}_{0}}}^{-1} \mathcal{A}_{2}{\widetilde{\mathcal{A}_{0}}}^{-1} \mathcal{A}_{1}{\widetilde{\mathcal{A}_{0}}}^{-1}\right)+O\left(\varepsilon^{4}\right) .
\end{aligned}
$$

It holds that ${\widetilde{\mathcal{A}_{0}}}^{-1} \Phi_{2}=\Phi_{1}+\Phi_{2}$ and $\left({\widetilde{\mathcal{A}_{0}}}^{-1}\right)^{*} \Phi_{3}=\Phi_{4}$. Moreover, using Lemma 3.9 we have

$$
\left(\widetilde{\mathcal{A}}_{0}^{-1} \mathcal{A}_{1}\right)^{*} \Phi_{3}=\mathcal{A}_{1}^{*} \Phi_{4}=\left(\begin{array}{c}
\mathcal{K}_{1,+}^{\mathbf{k}_{b}}\left[\mathbb{1}_{\partial D}\right] \\
0
\end{array}\right)=0
$$

As a consequence, (3.27) simplifies into

$$
\begin{aligned}
\left\langle\Phi_{3},\left(\widetilde{\mathcal{A}_{0}}+\mathcal{B}(\varepsilon)\right)^{-1} \Phi_{2}\right\rangle= & 1-\varepsilon^{2}\left\langle\Phi_{4}, \mathcal{A}_{2}\left(\Phi_{1}+\Phi_{2}\right)\right\rangle-\varepsilon^{3}\left\langle\Phi_{4}, \mathcal{A}_{3}\left(\Phi_{1}+\Phi_{2}\right)\right\rangle \\
& +\varepsilon^{3}\left\langle\Phi_{4}, \mathcal{A}_{2} \widetilde{\mathcal{A}_{0}}{ }^{-1} \mathcal{A}_{1}\left(\Phi_{1}+\Phi_{2}\right)\right\rangle+O\left(\varepsilon^{4}\right) .
\end{aligned}
$$


Let us evaluate the terms appearing in (3.28). First, it holds that

$$
\left\langle\Phi_{4}, \mathcal{A}_{2}\left(\Phi_{1}+\Phi_{2}\right)\right\rangle=\left\langle\mathbb{1}_{\partial D}, \mathcal{K}_{2,+}^{\mathbf{k}_{b}, *}\left[\psi_{0}^{+}\right]\right\rangle-\mu\left\langle\mathbb{1}_{\partial D},\left(\frac{1}{2}+\mathcal{K}_{+}^{*}\right) \psi_{0}^{+}\right\rangle .
$$

Recall that $\mathcal{K}_{+}^{*}\left[\psi_{0}^{+}\right]=\frac{1}{2} \psi_{0}^{+}$. By Lemma 3.9, we have

$$
\left\langle\mathcal{K}_{2,+}^{\mathbf{k}_{b}}\left[\mathbb{1}_{\partial D}\right], \psi_{0}^{+}\right\rangle=-k_{b}^{2} \int_{\partial D} \int_{D} G_{+}^{\mathbf{0}}(\mathbf{x}, \mathbf{y}) \psi_{0}^{+}(\mathbf{x}) \mathrm{d} \mathbf{y} \mathrm{d} \sigma(\mathbf{x})=-k_{b}^{2} \int_{D} \widetilde{S_{+}}\left[\psi_{0}^{+}\right](\mathbf{y}) \mathrm{d} \mathbf{y}=\frac{k_{b}^{2}|D|}{C_{D, \mathcal{R}}^{+}} .
$$

Hence,

$$
\left\langle\Phi_{4}, \mathcal{A}_{2}\left(\Phi_{1}+\Phi_{2}\right)\right\rangle=\frac{k_{b}^{2}|D|}{C_{D, \mathcal{R}}^{+}}-\mu=\mu_{M}-\mu,
$$

where $\mu_{M}$ was defined in (2.2). Similarly, using Lemma 3.9, one gets

$$
\left\langle\Phi_{4}, \mathcal{A}_{3}\left(\Phi_{1}+\Phi_{2}\right)\right\rangle=\left\langle\mathbb{1}_{\partial D}, \mathcal{K}_{3,+}^{\mathbf{k}_{b}, *}\left[\psi_{0}^{+}\right]\right\rangle=-k_{b}^{2} C_{1},
$$

where for simplicity we set

$$
C_{1}:=\int_{D} \int_{\partial D} G_{1,+}^{\mathbf{k}_{b}}(\mathbf{x}, \mathbf{y}) \psi_{0}^{+}(\mathbf{x}) \mathrm{d} \sigma(\mathbf{x}) \mathrm{d} \mathbf{y} .
$$

Finally, it remains to evaluate $\left\langle\Phi_{4}, \mathcal{A}_{2} \widetilde{\mathcal{A}}_{0}^{-1} \mathcal{A}_{1}\left(\Phi_{1}+\Phi_{2}\right)\right\rangle$. First, we have that

$$
\mathcal{A}_{1}\left[\Phi_{1}+\Phi_{2}\right]=\left(\begin{array}{c}
\left(\mathcal{S}_{1,+}^{\mathbf{k}_{b}}-\mathcal{S}_{1,+}^{\mathbf{k}}\right)\left[\psi_{0}^{+}\right] \\
\mathcal{K}_{1,+}^{\mathbf{k}_{b}, *}\left[\psi_{0}^{+}\right]
\end{array}\right) \text {. }
$$

By noticing that $\overline{k_{b}}=\bar{k}$, together with the expression (3.12), we see that the contribution of $\bar{k}$ in $\mathcal{S}_{1,+}^{\mathbf{k}_{b}}-\mathcal{S}_{1,+}^{\mathbf{k}}$ cancels. Hence,

$$
\left(\mathcal{S}_{1,+}^{\mathbf{k}_{b}}-\mathcal{S}_{1,+}^{\mathbf{k}}\right)\left[\psi_{0}^{+}\right]=\left(k_{b, d}-k_{d}\right) \int_{\partial D} \frac{-\mathrm{i} x_{d} y_{d}}{|\Gamma|} \psi_{0}^{+}(\mathbf{y}) \mathrm{d} \sigma(\mathbf{y})=\frac{-\mathrm{i} M_{1}}{|\Gamma|}\left(k_{b, d}-k_{d}\right) x_{d} .
$$

In particular,

$$
{\widetilde{\mathcal{A}_{0}}}^{-1} \mathcal{A}_{1}\left[\Phi_{1}+\Phi_{2}\right]=\left(\begin{array}{c}
\left(-\frac{1}{2}+\mathcal{K}_{+}^{*}+\Pi\right) \mathcal{K}_{1,+}^{\mathbf{k}_{b}, *}\left[\psi_{0}^{+}\right] \\
\left(-\frac{1}{2}+\mathcal{K}_{+}^{*}+\Pi\right)^{-1} \mathcal{K}_{1,+}^{\mathbf{k}_{b}, *}\left[\psi_{0}^{+}\right]-\frac{-\mathrm{i} M_{1}}{|\Gamma|}\left(k_{b, d}-k_{d}\right)\left(\psi_{1}^{+}-M_{1} C_{D, \mathcal{R}}^{+} \psi_{0}^{+}\right)
\end{array}\right)
$$

On the other hand, we have (using the fact that $\mathcal{K}_{+}\left[\mathbb{1}_{\partial D}\right]=\frac{1}{2} \mathbb{1}_{\partial D}$ )

$$
\mathcal{A}_{2}^{*} \Phi_{4}=\left(\begin{array}{c}
\mathcal{K}_{2,+}^{\mathbf{k}_{b}}\left[\mathbb{1}_{\partial D}\right] \\
-\mu\left(\frac{1}{2}+\mathcal{K}_{+}\right) \mathbb{1}_{\partial D}
\end{array}\right)=\left(\begin{array}{c}
-k_{b}^{2} \int_{D} G_{+}^{\mathbf{0}}(\mathbf{x}, \mathbf{y}) \mathrm{d} \mathbf{y} \\
-\mu \mathbb{1}_{\partial D}
\end{array}\right) .
$$

Altogether, we obtain that

$$
\left\langle\Phi_{4}, \mathcal{A}_{2}{\widetilde{\mathcal{A}_{0}}}^{-1} \mathcal{A}_{1}\left(\Phi_{1}+\Phi_{2}\right)\right\rangle=-k_{b}^{2}\left\langle\int_{D} G_{+}^{\mathbf{0}}(\mathbf{x}, \mathbf{y}) \mathrm{d} \mathbf{y},\left(-\frac{1}{2}+\mathcal{K}_{+}^{*}+\Pi\right)^{-1} \mathcal{K}_{1,+}^{\mathbf{k}_{b}, *}\left[\psi_{0}^{+}\right]\right)+\frac{\mathrm{i} \mu M_{1}^{2} C_{D, \mathcal{R}}^{+}}{|\Gamma|}\left(k_{b, d}-k_{d}\right) .
$$


Let us compute the inner product. We introduce the map $H: \bar{D} \rightarrow \mathbb{C}$ defined by

$$
\forall \mathbf{x} \in \bar{D}, \quad H(\mathbf{x}):=\int_{\partial D} G_{1,+}^{\mathbf{k}_{b}}(\mathbf{x}, \mathbf{y}) \psi_{0}^{+}(\mathbf{y}) \mathrm{d} \sigma(\mathbf{y}),
$$

and we set $T=\mathcal{S}_{+}^{-1}\left[\left.H\right|_{-}\right]$and $I=\int_{\partial D} T \mathrm{~d} \sigma$. Thanks to (3.14), we see that $\Delta H=0$ on $D$. Together with the jump relation formulae (see Lemma 3.4), we deduce that

$$
\left(-\frac{1}{2}+\mathcal{K}_{+}^{*}\right)[T]=\frac{\partial H}{\partial_{\nu_{\mathbf{x}}}}=\mathcal{K}_{1,+}^{\mathbf{k}_{b}, *}\left[\psi_{0}^{+}\right]
$$

Therefore

$$
\left(-\frac{1}{2}+\mathcal{K}_{+}^{*}+\Pi\right)^{-1} \mathcal{K}_{1,+}^{\mathbf{k}_{b}, *}\left[\psi_{0}^{+}\right]=T-I \psi_{0}^{+}
$$

It follows that

$$
\begin{gathered}
\left\langle\int_{D} G_{+}^{\mathbf{0}}(\mathbf{x}, \mathbf{y}) \mathrm{d} \mathbf{y},\left(-\frac{1}{2}+\mathcal{K}_{+}^{*}+\Pi\right)^{-1} \mathcal{K}_{1,+}^{\mathbf{k}_{b}, *}\left[\psi_{0}^{+}\right]\right)=\int_{D}\left(\int_{\partial D} G_{+}^{\mathbf{0}}(\mathbf{x}, \mathbf{y})\left(T-I \psi_{0}^{+}\right)(\mathbf{y}) \mathrm{d} \sigma(\mathbf{y})\right) \mathrm{d} \mathbf{x} \\
=\int_{D} \widetilde{\mathcal{S}_{+}}\left[T-I \psi_{0}^{+}\right](\mathbf{x}) \mathrm{d} \mathbf{x}=\int_{D} H(\mathbf{x}) \mathrm{d} \mathbf{x}+I \frac{|D|}{C_{D, \mathcal{R}}^{+}}=C_{1}+I \frac{|D|}{C_{D, \mathcal{R}}^{+}},
\end{gathered}
$$

where $C_{1}$ was defined in (3.29). It remains to compute the constant $I$. We have

$$
\begin{aligned}
I & =\left\langle\mathbb{1}_{\partial D}, T\right\rangle=\left\langle\mathbb{1}_{\partial D}, \mathcal{S}_{+}^{-1}[H]\right\rangle=\left\langle\mathcal{S}_{+}^{-1}\left[\mathbb{1}_{\partial D}\right], H\right\rangle=-C_{D, \mathcal{R}}^{+}\left\langle\psi_{0}^{+}, H\right\rangle \\
& =-C_{D, \mathcal{R}}^{+} \int_{\partial D} \int_{\partial D} G_{1,+}^{\mathbf{k}_{b}}(\mathbf{x}, \mathbf{y}) \psi_{0}^{+}(\mathbf{x}) \psi_{0}^{+}(\mathbf{y}) \mathrm{d} \sigma(\mathbf{x}) \mathrm{d} \sigma(\mathbf{y}) \\
& =\frac{-C_{D, \mathcal{R}}^{+}}{2} \int_{\partial D} \int_{\partial D} \psi_{0}^{+}(\mathbf{x}) \psi_{0}^{+}(\mathbf{y})\left(G_{1,+}^{\mathbf{k}_{b}}(\mathbf{x}, \mathbf{y})+G_{1,+}^{\mathbf{k}_{b}}(\mathbf{y}, \mathbf{x})\right) \mathrm{d} \sigma(\mathbf{x}) \mathrm{d} \sigma(\mathbf{y}),
\end{aligned}
$$

where we performed the change of variable $(\mathbf{x}, \mathbf{y}) \rightarrow(\mathbf{y}, \mathbf{x})$ to obtain the last equality. From the expression of $G_{1,+}^{\mathbf{k}_{b}}$ in (3.12) and the symmetry relation (3.13), we get

$$
I=\frac{\mathrm{i} C_{D, \mathcal{R}}^{+}}{|\Gamma|} k_{b, d} \int_{\partial D} x_{d} \psi_{0}(\mathbf{x}) \mathrm{d} \sigma(\mathbf{x}) \int_{\partial D} y_{d} \psi_{0}(\mathbf{y}) \mathrm{d} \sigma(\mathbf{y})=\frac{\mathrm{i} M_{1}^{2} C_{D, \mathcal{R}}^{+}}{|\Gamma|} k_{b, d} .
$$

Altogether, the above calculations yield

$$
\begin{aligned}
1-\left\langle\Phi_{3},\left(\widetilde{\mathcal{A}_{0}}+\mathcal{B}(\varepsilon)\right)^{-1} \Phi_{2}\right\rangle_{\mathcal{H}^{--}}= & \varepsilon^{2}\left(\mu_{M}-\mu\right)+\varepsilon^{3}\left(-k_{b}^{2} C_{1}\right) \\
& -\varepsilon^{3}\left(-k_{b}^{2}\left[C_{1}+\frac{\mathrm{i} M_{1}^{2}|D|}{|\Gamma|} k_{b, d}\right]+\frac{\mathrm{i} \mu M_{1}^{2} C_{D, \mathcal{R}}^{+}}{|\Gamma|}\left(k_{b, d}-k_{d}\right)\right)+O\left(\varepsilon^{4}\right) \\
= & \varepsilon^{2}\left(\mu_{M}-\mu\right)+\varepsilon^{3} \mathrm{i} \frac{M_{1}^{2}}{|\Gamma|}\left(k_{b}^{2} k_{b, d}|D|-\mu\left(k_{b, d}-k_{d}\right) C_{D, \mathcal{R}}^{+}\right)+O\left(\varepsilon^{4}\right) \\
= & \varepsilon^{2}\left(\mu_{M}-\mu\right)\left(1+\varepsilon \mathrm{i} \frac{M_{1}^{2} k_{b, d} C_{D, \mathcal{R}}^{+}}{|\Gamma|}\right)+\varepsilon^{3} \mathrm{i} \mu \frac{M_{1}^{2} k_{d} C_{D, \mathcal{R}}^{+}}{|\Gamma|}+O\left(\varepsilon^{4}\right) .
\end{aligned}
$$

- Evaluation of $\left\langle\Phi_{3},\left(\widetilde{\mathcal{A}_{0}}+\mathcal{B}(\varepsilon)\right)^{-1} F(\varepsilon)\right\rangle$. From (3.22), we obtain that

$$
F=\varepsilon F_{1}+\varepsilon^{2} F_{2}+\varepsilon^{3} F_{3}+O\left(\varepsilon^{4}\right),
$$


with

$F_{1}:=-2 \mathrm{i} u_{0}\left(\begin{array}{c}k_{d} x_{d} \\ 0\end{array}\right), \quad F_{2}:=-2 \mathrm{i} u_{0}\left(\begin{array}{c}-\mathrm{i}(\bar{k} \cdot \bar{x}) k_{d} x_{d} \\ 0\end{array}\right), \quad$ and $\quad F_{3}:=-2 \mathrm{i} u_{0}\left(\begin{array}{c}\frac{1}{6}\left(k_{d} x_{d}\right)^{3}-\frac{1}{2}(\bar{k} \cdot \bar{x})^{2} k_{d} x_{d} \\ \mu k_{d} \nu_{d}\end{array}\right)$.

Using the decomposition (3.27) and similar estimates as before, we get

$$
\begin{aligned}
&\left\langle\Phi_{3},\left(\widetilde{\mathcal{A}_{0}}+\mathcal{B}(\varepsilon)\right)^{-1} F(\varepsilon)\right\rangle=\varepsilon\left\langle\Phi_{3},{\widetilde{\mathcal{A}_{0}}}^{-1} F_{1}\right\rangle+\varepsilon^{2}\left\langle\Phi_{3},{\widetilde{\mathcal{A}_{0}}}^{-1} F_{2}\right\rangle \\
&-\varepsilon^{3}\left\langle\Phi_{3},{\widetilde{\mathcal{A}_{0}}}^{-1} \mathcal{A}_{2}{\widetilde{\mathcal{A}_{0}}}^{-1} F_{1}\right\rangle+\varepsilon^{3}\left\langle\Phi_{3},{\widetilde{\mathcal{A}_{0}}}^{-1} F_{3}\right\rangle+O\left(\varepsilon^{4}\right) .
\end{aligned}
$$

We have that $\left\langle\Phi_{3}, \widetilde{\mathcal{A}}_{0}^{-1} F_{1}\right\rangle=\left\langle\Phi_{3}, \widetilde{\mathcal{A}}_{0}^{-1} F_{2}\right\rangle=\left\langle\Phi_{3}, \widetilde{\mathcal{A}}_{0}{ }^{-1} F_{3}\right\rangle=0$. Also, using (3.30), we have $\left\langle\Phi_{3},{\widetilde{\mathcal{A}_{0}}}^{-1} \mathcal{A}_{2}{\widetilde{\mathcal{A}_{0}}}^{-1} F_{1}\right\rangle=\left\langle\Phi_{4}, \mathcal{A}_{2}{\widetilde{\mathcal{A}_{0}}}^{-1} F_{1}\right\rangle=-2 \mathrm{i} u_{0} k_{d} \mu\left\langle\mathbb{1}_{\partial D}, \psi_{1}^{+}-M_{1} C_{D, \mathcal{R}}^{+} \psi_{0}^{+}\right\rangle=2 \mathrm{i} u_{0} k_{d} \mu M_{1} C_{D, \mathcal{R}}^{+}$

We can conclude that

$$
\left\langle\Phi_{3},\left(\widetilde{\mathcal{A}_{0}}+\mathcal{B}(\varepsilon)\right)^{-1} F(\varepsilon)\right\rangle=-\varepsilon^{3} 2 \mathrm{i} u_{0} k_{d} \mu M_{1} C_{D, \mathcal{R}}^{+}+O\left(\varepsilon^{4}\right) .
$$

- Evaluation of $\alpha$. From the above calculations, we deduce that

$$
\alpha=-\frac{\varepsilon 2 \mathrm{i} u_{0} \mu k_{d} M_{1} C_{D, \mathcal{R}}^{+}+O\left(\varepsilon^{2}\right)}{\left(\mu_{M}-\mu\right)\left(1+\varepsilon \mathrm{i} \frac{M_{1}^{2} k_{b, d} C_{D, \mathcal{R}}^{+}}{|\Gamma|}\right)+\varepsilon \mathrm{i} \mu \frac{M_{1}^{2} k_{d} C_{D, \mathcal{R}}^{+}}{|\Gamma|}+O\left(\varepsilon^{2}\right)} .
$$

In order to simplify this expression, we recall the scattering function $g_{s}(\mu, \varepsilon)$ defined in (2.3). We can check that

$$
\alpha=\left(2 \mathrm{i} u_{0} k_{d} C_{D, \mathcal{R}}^{+}+O(\varepsilon)\right) \cdot g_{s}(\mu, \varepsilon),
$$

in the sense

$$
\exists C \in \mathbb{R}^{+}, \forall \varepsilon \geq 0, \forall \mu>0, \quad\left|\alpha-\left(2 \mathrm{i} u_{0} k_{d}\right) C_{D, \mathcal{R}}^{+} g_{s}(\mu, \varepsilon)\right| \leq C \varepsilon\left|g_{s}(\mu, \varepsilon)\right| .
$$

- Evaluation of $\Psi_{-}$. We finally calculate $\Psi_{-}$defined in the second equation of (3.26). Using similar calculations as before, we get

$$
\begin{aligned}
\Psi_{-} & =\varepsilon{\widetilde{\mathcal{A}_{0}}}^{-1} F_{1}-\alpha \Phi_{1}+\alpha{\widetilde{\mathcal{A}_{0}}}^{-1} \Phi_{2}+O\left(\varepsilon^{2}+|\varepsilon \alpha|\right) \\
& =\left(2 \mathrm{i} u_{0} k_{d}\right)\left(\varepsilon\left(\begin{array}{c}
0 \\
\psi_{1}^{+}-M_{1} C_{D, \mathcal{R}}^{+} \psi_{0}^{+}
\end{array}\right)+g_{s}(\mu, \varepsilon) C_{D, \mathcal{R}}^{+}\left(\begin{array}{c}
0 \\
\psi_{0}^{+}
\end{array}\right)\right)+O\left(\varepsilon\left|g_{s}(\mu, \varepsilon)\right|\right) .
\end{aligned}
$$

\subsection{Microscopic scattered field}

Recall (3.19), we have

$$
u_{\varepsilon}(\mathbf{x})=-2 \mathrm{i} u_{0} \sin \left(\varepsilon k_{d} x_{d}\right) \mathrm{e}^{-\mathrm{i} \varepsilon \bar{k} \cdot \bar{x}}+u_{\varepsilon}^{s}(\mathbf{x}),
$$

where $u_{\varepsilon}^{s}=\widetilde{\mathcal{S}_{+}^{\varepsilon k}}\left[\psi_{\varepsilon}\right]$ is the scattered field. Note that $\Psi_{+}$do not contribute to the field outside the bubbles. Using (3.31), we obtain that

$$
u_{\varepsilon}^{s}(\mathbf{x})=\left(2 \mathrm{i} u_{0} k_{d}\right) \int_{D} G_{+}^{\varepsilon \mathbf{k}}(\mathbf{x}, \mathbf{y})\left(\varepsilon\left[\psi_{1}^{+}-M_{1} C_{D, \mathcal{R}}^{+} \psi_{0}^{+}\right]+g_{s}(\mu, \varepsilon) C_{D, \mathcal{R}}^{+} \psi_{0}^{+}\right)(\mathbf{y}) \mathrm{d} \sigma(\mathbf{y})+O\left(\varepsilon\left|g_{s}(\mu, \varepsilon)\right|\right) .
$$


We now simply the above integral by exploiting decomposition of $G_{+}^{\varepsilon \mathbf{k}}$. Recall that $L>0$ is chosen such that, for all $\mathbf{y} \in D$, it holds that $0 \leq y_{d} \leq L$. Together with (3.10), we obtain that, for $x_{d} \geq L$, we have $G_{+}^{\mathbf{k}}=G_{+, p}^{\mathbf{k}}+G_{+, e}^{\mathbf{k}}$, where

$$
\begin{aligned}
& G_{+, p}^{\mathbf{k}}(\mathbf{x}, \mathbf{y})=\left(\frac{\mathrm{e}^{\mathrm{i} k_{d}\left(x_{d}-y_{d}\right)}}{2 \mathrm{i} k_{d}|\Gamma|}-\frac{\mathrm{e}^{\mathrm{i} k_{d}\left(x_{d}+y_{d}\right)}}{2 \mathrm{i} k_{d}|\Gamma|}\right) \mathrm{e}^{-\mathrm{i} \bar{k} \cdot \bar{x}}=-\frac{\sin \left(k_{d} y_{d}\right)}{k_{d}|\Gamma|} \mathrm{e}^{\mathrm{i}\left(k_{d} x_{d}-\bar{k} \cdot \bar{x}\right)} \\
& G_{+, e}^{\mathbf{k}}(\mathbf{x}, \mathbf{y})=-\sum_{\ell \in \mathcal{R}^{*} \backslash\{\mathbf{0}\}}\left(\frac{\mathrm{e}^{\mathrm{i}(\ell-\bar{k}) \cdot(\bar{x}-\bar{y})}}{|\Gamma| \sqrt{|\ell-\bar{k}|^{2}-k^{2}}} \sinh \left(\sqrt{|\ell-\bar{k}|^{2}-k^{2}} y_{d}\right)\right) \mathrm{e}^{-\sqrt{|\ell-\bar{k}|^{2}-k^{2}} x_{d}}
\end{aligned}
$$

It is clear that $G_{+, p}^{\mathbf{k}}$ consists of the propagative mode, while $G_{+, e}^{\mathbf{k}}$ consists of the evanescent modes which are exponentially decaying away from the plane.

Accordingly, we write $\widetilde{\mathcal{S}_{+}^{\mathbf{k}}}=\widetilde{\mathcal{S}_{+, p}^{\mathbf{k}}}+\widetilde{\mathcal{S}_{+}^{\mathbf{k}}, e}$, with

$$
\begin{aligned}
& \widetilde{\mathcal{S}_{+, p}^{\mathbf{k}}}[\psi](\mathbf{x})=\int_{\partial D} G_{+, p}^{\mathbf{k}}(\mathbf{x}, \mathbf{y}) \psi(\mathbf{y}) \mathrm{d} \sigma(\mathbf{y}), \\
& \widetilde{\mathcal{S}_{+, e}^{\mathbf{k}}}[\psi](\mathbf{x})=\int_{\partial D} G_{+, e}^{\mathbf{k}}(\mathbf{x}, \mathbf{y}) \psi(\mathbf{y}) \mathrm{d} \sigma(\mathbf{y}) .
\end{aligned}
$$

In particular, in the case when $\mathbf{k}=0$, we can deduce using (3.2) that

$$
\begin{aligned}
& \widetilde{S_{+, p}^{\mathbf{k}=0}}[\psi](\mathbf{x})=-\frac{1}{|\Gamma|} \int_{\partial D} y_{d} \psi(\mathbf{y}) \mathrm{d} \sigma(\mathbf{y}), \\
& \widetilde{S_{+, e}^{\mathbf{k}=\mathbf{0}}}[\psi](\mathbf{x})=\widetilde{S_{+}}[\psi](\mathbf{x})+\frac{1}{|\Gamma|} \int_{\partial D} y_{d} \psi(\mathbf{y}) \mathrm{d} \sigma(\mathbf{y}),
\end{aligned}
$$

for $\psi \in H^{-1 / 2}$ and $x_{d} \geq L$.

Following (3.5) and (3.10), we set

$$
\widetilde{S_{+, p, 0}^{\mathbf{k}}}[\psi](\mathbf{x}):=-\mathrm{e}^{\mathrm{i}\left(k_{d} x_{d}-\bar{k} \cdot \bar{x}\right)} \frac{1}{|\Gamma|} \int_{\partial D} y_{d} \psi(\mathbf{y}) \mathrm{d} \sigma(\mathbf{y}) .
$$

and

$$
\widetilde{S_{+, e, 0}^{\mathbf{k}}}[\psi](\mathbf{x}):=\mathrm{e}^{-\mathrm{i} \bar{k} \cdot \bar{x}}\left(\widetilde{S_{+}}[\psi](\mathbf{x})+\frac{1}{|\Gamma|} \int_{\partial D} y_{d} \psi(\mathbf{y}) \mathrm{d} \sigma(\mathbf{y})\right) .
$$

The operators $\widetilde{S_{+, p, 0}^{\varepsilon \mathbf{k}}}$ and $\widetilde{S_{+, e, 0}^{\varepsilon \mathbf{k}}}$ are good approximations for $\widetilde{S_{+, p}^{\varepsilon \mathbf{k}}}$ and $\widetilde{S_{+, e}^{\varepsilon \mathbf{k}}}$ respectively. Actually, from the expression of $G_{+, p}^{\mathbf{k}}$ and $G_{+, e}^{\mathbf{k}}$, we have the following results.

Lemma 3.10. There exists $C \in \mathbb{R}^{+}$such that, for all $\varepsilon \geq 0$, all $x_{d} \geq L$ and all $\psi \in H^{-1 / 2}$, it holds that

$$
\left|\left(\widetilde{S_{+, p}^{\varepsilon \mathbf{k}}}-\widetilde{S_{+, p, 0}^{\varepsilon \mathbf{k}}}\right)[\psi]\right|(\mathbf{x})+\frac{1}{\varepsilon}\left|\nabla\left(\widetilde{S_{+, p}^{\varepsilon \mathbf{k}}}-\widetilde{S_{+, p, 0}^{\varepsilon \mathbf{k}}}\right)[\psi]\right|(\mathbf{x}) \leq C\|\psi\|_{H^{-1 / 2}} \varepsilon^{2}
$$

and that

$$
\left|\left(\widetilde{S_{+, e}^{\varepsilon \mathbf{k}}}-\widetilde{S_{+, e, 0}^{\varepsilon \mathbf{k}}}\right)[\psi]\right|(\mathbf{x})+\frac{1}{\varepsilon}\left|\nabla\left(\widetilde{S_{+, e}^{\varepsilon \mathbf{k}}}-\widetilde{S_{+, e, 0}^{\varepsilon \mathbf{k}}}\right)[\psi]\right|(\mathbf{x}) \leq C\|\psi\|_{H^{-1 / 2}} \varepsilon .
$$

Now, we determine the functions $\alpha_{1}$ and $\alpha_{2}$ defined in (2.4)-(2.5). 
Lemma 3.11. The function $-C_{D, \mathcal{R}}^{+} \widetilde{S_{+}}\left[\psi_{0}^{+}\right]$is the solution to (2.4), while the function $\widetilde{S_{+}}\left[\psi_{1}^{+}-\right.$ $\left.M_{1} C_{D, \mathcal{R}}^{+} \psi_{0}^{+}\right]$is the solution to (2.5), i.e.

$$
-C_{D, \mathcal{R}}^{+} \widetilde{\mathcal{S}_{+}}\left[\psi_{0}^{+}\right]=\alpha_{0} \quad \text { and } \quad \widetilde{S_{+}}\left[\psi_{1}^{+}-M_{1} C_{D, \mathcal{R}}^{+} \psi_{0}^{+}\right]=\alpha_{1}
$$

In particular, from (3.36) and the fact that $G_{+, e}^{\mathbf{k}=\mathbf{0}}$ is exponentially decaying, it holds that

$$
\alpha_{0, \infty}=-\frac{1}{|\Gamma|} \int_{\partial D} y_{d}\left(-C_{D, \mathcal{R}}^{+} \psi_{0}^{+}\right)=\frac{M_{1} C_{D, \mathcal{R}}^{+}}{|\Gamma|}
$$

and

$$
\alpha_{1, \infty}=-\frac{1}{|\Gamma|} \int_{\partial D} y_{d}\left(\psi_{1}^{+}-M_{1} C_{D, \mathcal{R}}^{+} \psi_{0}^{+}\right)=\frac{-\left\langle y_{d}, \psi_{1}^{+}\right\rangle}{|\Gamma|}+\frac{M_{1}^{2} C_{D, \mathcal{R}}^{+}}{|\Gamma|} .
$$

Finally, we are ready to prove our main result Theorem 2.1.

\section{- Proof of Theorem 2.1}

We introduce

$$
\begin{aligned}
u_{1}^{\varepsilon}(\mathbf{x}) & :=\left(2 \mathrm{i} u_{0} k_{d}\right) \widetilde{S_{+, p, 0}^{\varepsilon \mathbf{k}}}\left[\varepsilon\left(\psi_{1}^{+}-M_{1} C_{D, \mathcal{R}}^{+} \psi_{0}^{+}\right)+g_{s}(\mu, \varepsilon)\left(C_{D, \mathcal{R}}^{+} \psi_{0}^{+}\right)\right](\mathbf{x}) \\
& =\left(2 \mathrm{i} u_{0} k_{d}\right) \mathrm{e}^{\mathrm{i} \varepsilon\left(k_{d} x_{d}-\bar{k} \cdot \bar{x}\right)}\left(\varepsilon \alpha_{1, \infty}-g_{s}(\mu, \varepsilon) \alpha_{0, \infty}\right)
\end{aligned}
$$

and

$$
\begin{aligned}
u_{\mathrm{BL}}^{\varepsilon}(\mathbf{x}) & :=\left(2 \mathrm{i} u_{0} k_{d}\right) \widetilde{S_{+, e, 0}^{\varepsilon \mathbf{k}}}\left[\varepsilon\left(\psi_{1}^{+}-M_{1} C_{D, \mathcal{R}}^{+} \psi_{0}^{+}\right)+g_{s}(\mu, \varepsilon)\left(C_{D, \mathcal{R}}^{+} \psi_{0}^{+}\right)\right](\mathbf{x}) \\
& =\left(2 \mathrm{i} u_{0} k_{d}\right) \mathrm{e}^{-\mathrm{i} \varepsilon \bar{k} \cdot \bar{x}}\left[\varepsilon\left(\alpha_{1}(\mathbf{x})-\alpha_{1, \infty}\right)-g_{s}(\mu, \varepsilon)\left(\alpha_{0}(\mathbf{x})-\alpha_{0, \infty}\right)\right] .
\end{aligned}
$$

By (3.32) and Lemma 3.10, we deduce that there exists $C \geq 0$ such that,

$$
\left\|u_{\varepsilon}^{s}-\left(u_{1}^{\varepsilon}+u_{\mathrm{BL}}^{\varepsilon}\right)\right\|_{L^{\infty}\left(S_{L}\right)}+\frac{1}{\varepsilon}\left\|\nabla\left[u_{\varepsilon}^{s}-\left(u_{1}^{\varepsilon}+u_{\mathrm{BL}}^{\varepsilon}\right)\right]\right\|_{L^{\infty}\left(S_{L}\right)} \leq C\left(\varepsilon^{2}+\varepsilon\left|g_{s}(\mu, \varepsilon)\right|\right) .
$$

Going back to the macroscopic variable $\mathbf{X}=\varepsilon \mathbf{x}$ we can conclude the proof of Theorem 2.1.

\section{Numerical illustrations}

In this section, all the numerical results are obtained for the two-dimensional case. The bubbles are set along a lattice $a \mathbb{Z}$. We follow the approach taken in [5], in which the resonant frequencies, for both the one bubble and two bubble cases, were determined numerically. We calculate the characteristic value $\omega_{c}^{+}$of the block operator matrix $\mathcal{A}(3.21)$ directly. We then use this result to confirm the formula (2.6) for the periodic bubble resonance:

$$
\omega_{M}^{+}:=\sqrt{\frac{\delta C_{D, \mathcal{R}}^{+}}{|D|}} v_{b}
$$




\subsection{Implementation details}

Determination of $\omega_{c}^{+}$requires the calculation of the periodic Green function for the Helmholtz equation. It is well known that this function, the solution to (3.1), suffers from extremely slow convergence. It can be written in the form

$$
G_{\sharp}^{\mathbf{k}}(\mathbf{x}, \mathbf{y})=-\frac{i}{4} \sum_{n \in \mathbb{Z}} e^{i \bar{k} n a} H_{0}^{(1)}\left(k P_{n}\right),
$$

where $H_{0}^{(1)}$ is the Hankel function of the first kind of order zero, $P_{n}:=\sqrt{(\bar{x}-\bar{y}-n a)^{2}+\left(x_{d}-y_{d}\right)^{2}}$, and $\bar{k}=k \cos (\theta)$ is the component of the wave incident at angle $\theta$ along the $\bar{x}$ direction. The terms of the summation are of the order $O\left(n^{-\frac{1}{2}}\right)$ for large $n$, which makes the function computationally challenging.

In order to accelerate the convergence, we implement Ewald's method [18, 15], tailored to two-dimensional problems featuring one-dimensional periodicity. The periodic Green function is split into two components: a spatial component $G_{\sharp, s p a t}$ and a spectral component $G_{\sharp, \text { spec }}$ :

$$
G_{\sharp}^{\mathbf{k}}=G_{\sharp, \text { spat }}^{\mathbf{k}}+G_{\sharp, \text { spec }}^{\mathbf{k}},
$$

where

$$
G_{\sharp, \text { spat }}^{\mathbf{k}}(\mathbf{x}, \mathbf{y})=\frac{1}{4 \pi} \sum_{n \in \mathbb{Z}} e^{-i \bar{k} n a} \sum_{q=0}^{\infty}\left(\frac{k}{2 \mathcal{E}}\right)^{2 q} \frac{1}{q !} E_{q+1}\left(P_{n}^{2} \mathcal{E}^{2}\right),
$$

and

$$
\begin{aligned}
G_{\sharp, \text { spec }}^{\mathbf{k}}(\mathbf{x}, \mathbf{y}) & =\frac{1}{4 a} \sum_{p \in \mathbb{Z}} \frac{e^{-i k_{1 p}(\bar{x}-\bar{y})}}{i k_{2 p}} \times\left[e^{i k_{2 p}\left|x_{d}-y_{d}\right|} \operatorname{erfc}\left(\frac{i k_{2 p}}{2 \mathcal{E}}+\left|x_{d}-y_{d}\right| \mathcal{E}\right)\right. \\
& \left.+e^{-i k_{2 p}\left|x_{d}-y_{d}\right|} \operatorname{erfc}\left(\frac{i k_{2 p}}{2 \mathcal{E}}-\left|x_{d}-y_{d}\right| \mathcal{E}\right)\right] .
\end{aligned}
$$

Here, we set

$$
k_{1 p}=\bar{k}+\frac{2 \pi p}{a}, \quad \text { and } \quad k_{2 p}=\sqrt{k^{2}-k_{1 p}^{2}} .
$$

$k_{1 p}$ and $k_{2 p}$ are the Floquet wavenumbers along the $\bar{x}$ and $x_{d}$ directions, respectively. $E_{i}$ is the exponential integral. $\mathcal{E}$ is the splitting parameter, which we choose to be $\sqrt{\pi} / d$, an appropriate choice in a low frequency setting, and one which results in exponential convergence in both the $G_{\sharp, \text { spat }}$ and $G_{\sharp \text { spec }}$ terms. Due to the exponential convergence of the expressions in (4.1) and (4.2), we approximate the infinite sums with $n \in[-5,5], q \in[-15,15]$, and $p \in[-5,5]$. We apply corrections to account for periodicity to the usual singular diagonal terms of the discretized operators $\mathcal{S}_{+}^{\mathbf{k}}$ and $\mathcal{K}_{+}^{\mathbf{k}, *}$ that comprise the block operator matrix $\mathcal{A}$.

\subsection{Regime description}

In order to perform the calculations in the appropriate regime, which requires a significant contrast in both the bulk modulii, and the density, of the liquid and the bubbles, we take $\kappa_{b}$ and $\rho_{b}$ to be of order 1 , and $\kappa$ and $\rho$ to be of order $10^{3}$. The wave speeds inside and outside the bubbles are both of order 1 . 


$\begin{array}{cc}r & \omega_{M}^{+} \\ 0.1000 & 0.3898 \\ 0.3250 & 0.1191 \\ 0.5500 & 0.0694 \\ 0.7750 & 0.0483 \\ 1.0000 & 0.0366\end{array}$

Table 1: The resonant frequencies $\omega_{M}^{+}$obtained with the formula for a set of radii in the interval $[0.1,1]$. The spectrum of the corresponding block operator matrix $\mathcal{A}$ from Equation (3.21), which was calculated explicitly, is shown in Figure 1.

\subsection{Validation of the periodic resonant frequency formula}

We begin by calculating the resonant frequency given by the formula. In order to obtain the capacity $C_{D, \mathcal{R}}^{+}$, we first compute the eigenfunction $\psi$ corresponding to the eigenvalue $\frac{1}{2}$ for the operator $\mathcal{K}_{+}^{*}=\mathcal{K}_{+}^{0, *}$. We approximate a basis for the discretized version of this operator (and also for the single layer potential) with the family of functions having a value of 1 at a particular point, and 0 everywhere else. We fix the period to be $a=10$, and take a set of bubble radii in the range $r \in[0.1,1]$. The resonant frequencies obtained with the formula are given in Table 1. The characteristic values of $\mathcal{A}$ are shown in Figure 1. It is clear that the characteristic values $\omega_{c}^{+}$correspond to the resonant frequencies $\omega_{M}^{+}$obtained with the formula, confirming its validity.

\subsection{Effect of periodicity and bubble radii on resonance}

Let $\beta$ be the distance from the bubble centers to the reflective plane $\partial D$. In Figure 2, we fix the bubble radii at $r=1$, and analyze the relationship between periodicity and the resonant frequency, as we increase $\beta$, moving the bubbles further from the reflective plane. We find that $\omega_{M}^{+}$has a logarithmic dependency on the period, and is inversely proportional to the distance from the plane.

Similarly in Figure 3, where this time we fix the period at $a=5$, and consider the relationship between the bubble radii and the resonant frequency as the distance from the plane varies. Although resonant frequencies of bubbles are known to be inversely proportional to their radii, here we find that when the bubble radii are increased such that the bubbles are almost touching the reflective plane, the resonant frequency $\omega_{M}^{+}$in fact increases as we further increase the radii.

\section{References}

[1] T. Abboud and H. Ammari. Diffraction at a curved grating: TM and TE cases, Homogenization. J. Math. Anal. Appl., 202(3):995 - 1026, 1996.

[2] Y. Achdou, O. Pironneau, and F. Valentin. Effective boundary conditions for laminar flows over periodic rough boundaries. J. Comput. Phys., 147(1):187-218, 1998.

[3] G. Allaire and M. Amar. Boundary layer tails in periodic homogenization. ESAIM: COCV , 4:209-243, 1999. 


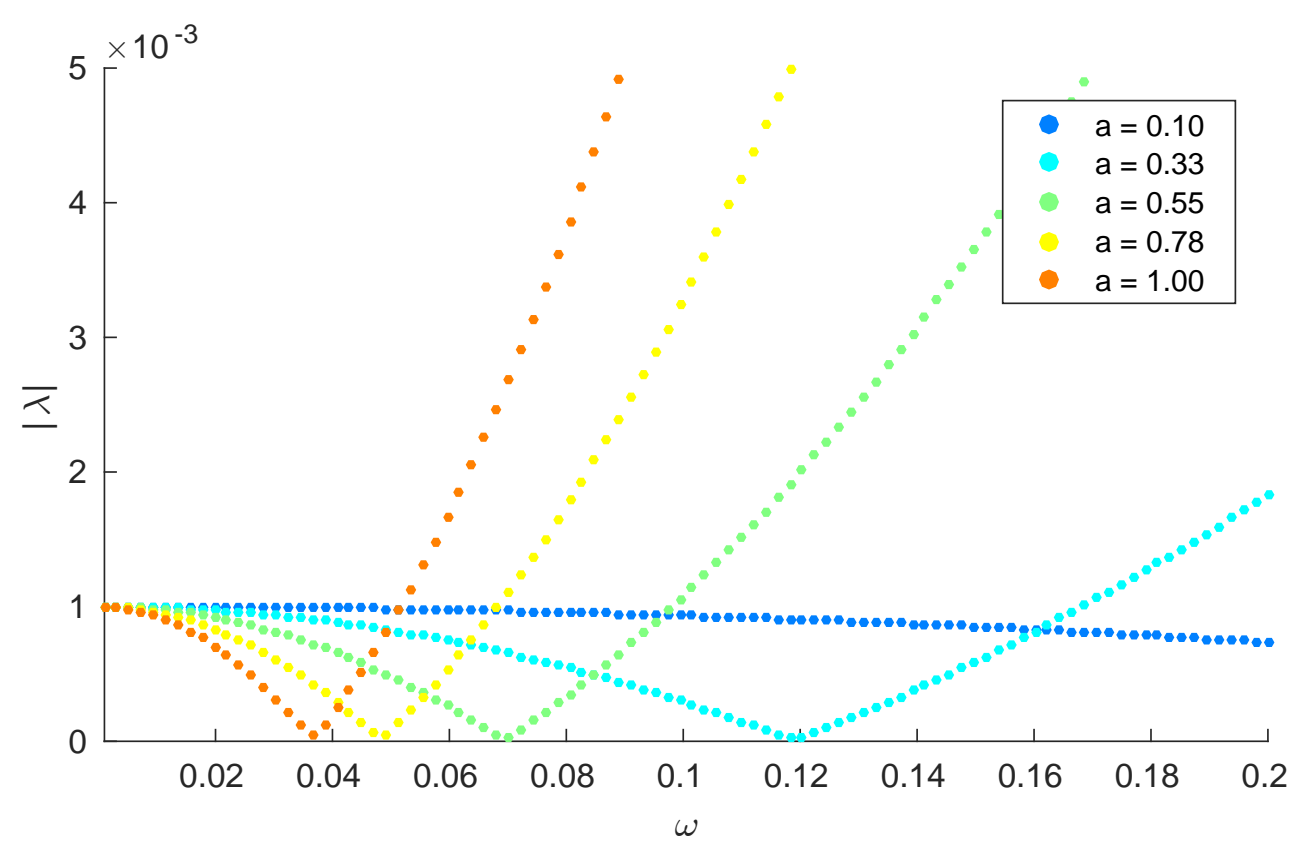

Figure 1: The smallest eigenvalue in the spectrum of the block operator matrix $\mathcal{A}$ from Equation (3.21), for a period of $a=10$, and a set of bubble radii in the interval $[0.1,1]$. We have characteristic values $\omega_{c}^{+}$where the eigenvalues go to 0 , indicating resonance. The characteristic values correspond to the resonant frequencies $\omega_{M}^{+}$, given in Table 1 , obtained with the formula for the same parameters.

[4] H. Ammari, Y. Deng, and P. Millien. Surface plasmon resonance of nanoparticles and applications in imaging. Archive for Rational Mechanics and Analysis, 220(1):109-153, 2016.

[5] H. Ammari, B. Fitzpatrick, D. Gontier, H. Lee, and H. Zhang. Minnaert resonances for acoustic waves in bubbly media. arXiv:1603.03982, 2016.

[6] H. Ammari, H. Kang, and H. Lee. Layer potential techniques in spectral analysis, volume 153. American Mathematical Society Providence, 2009.

[7] H. Ammari, P. Millien, M. Ruiz, and H. Zhang. Mathematical analysis of plasmonic nanoparticles: the scalar case. arXiv:1506.00866, 2015.

[8] H. Ammari, M. Ruiz, S. Yu, and H. Zhang. Mathematical analysis of plasmonic resonances for nanoparticles: the full Maxwell equations. Journal of Differential Equations, 261, 3615-3669, 2016.

[9] H. Ammari, M. Ruiz, W. Wu, S. Yu, and H. Zhang. Mathematical and numerical framework for metasurfaces using thin layers of periodically distributed plasmonic nanoparticles. Proceedings of the Royal Society A., 2016, to appear (arXiv:1602.05019).

[10] K. Ando and H. Kang. Analysis of plasmon resonance on smooth domains using spectral properties of the Neumann-Poincaré operator. J. Math. Anal. Appl., 435, 162-178, 2016. 


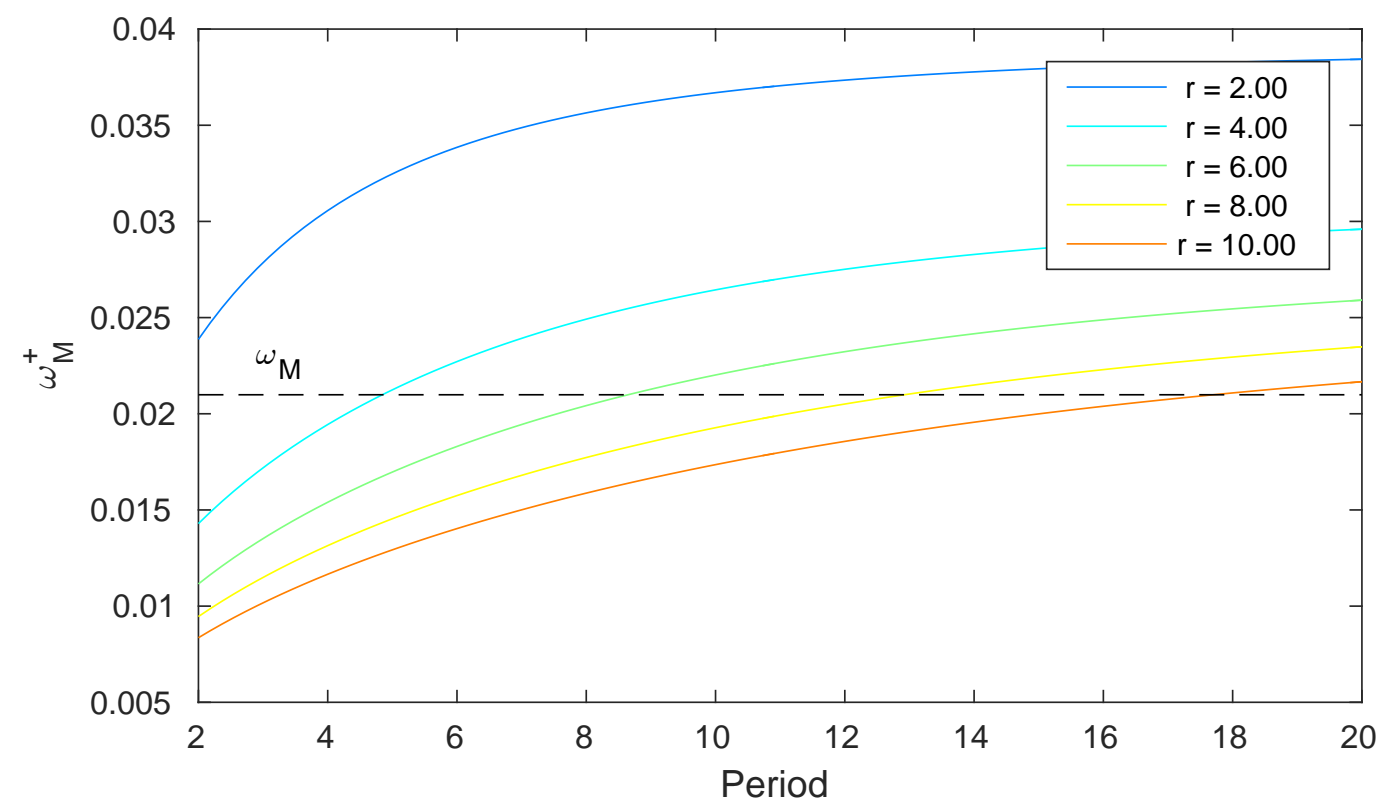

Figure 2: The bubble radii are fixed at $r=1$, and the distance from the bubble centers to the reflective plane $\partial D$ is represented by $\beta . \omega_{M}^{+}$has a logarithmic dependency on the period $a$, and decreases as the distance $r$ from the reflective plane $\partial D$ is increased. The one bubble resonant frequency $\omega_{M}$, for a bubble of radius 1 in free space, is plotted for reference.

[11] K. Ando, H. Kang, and H. Liu. Plasmon resonance with finite frequencies: a validation of the quasi-static approximation for diametrically small inclusions. SIAM J. Appl. Math., 76, 731-749, 2016.

[12] G. Bouchitté and D. Felbacq. Homogenization near resonances and artificial magnetism from dielectrics. C. R. Acad. Sci. Paris, 339(5):377-382, 2004.

[13] M. Briane and L. Pater. Homogenization of high-contrast two-phase conductivities perturbed by a magnetic field. Comparison between dimension two and dimension three. $J$. Math. Anal. Appl., 393(2):563-589, 2012.

[14] M. Camar-Eddine and L. Pater. Homogenization of high-contrast and non symmetric conductivities for non periodic columnar structures. Netw. Heterog. Media, 8:913 - 941, 2013.

[15] F. Capolino, D.R. Wilton, and W.A. Johnson. Efficient computation of the 2-d green's function for 1-d periodic structures using the ewald method. IEEE Transactions on antennas and propagation, 53(9):2977 - 2984, 2005.

[16] R.E. Collin. Field theory of guided waves. McGraw-Hill, 1960.

[17] M. Devaud, Th. Hocquet, J.-C. Bacri, and V. Leroy. The Minnaert bubble: an acoustic approach. Eur. J. Phys., 29(6):1263, 2008. 


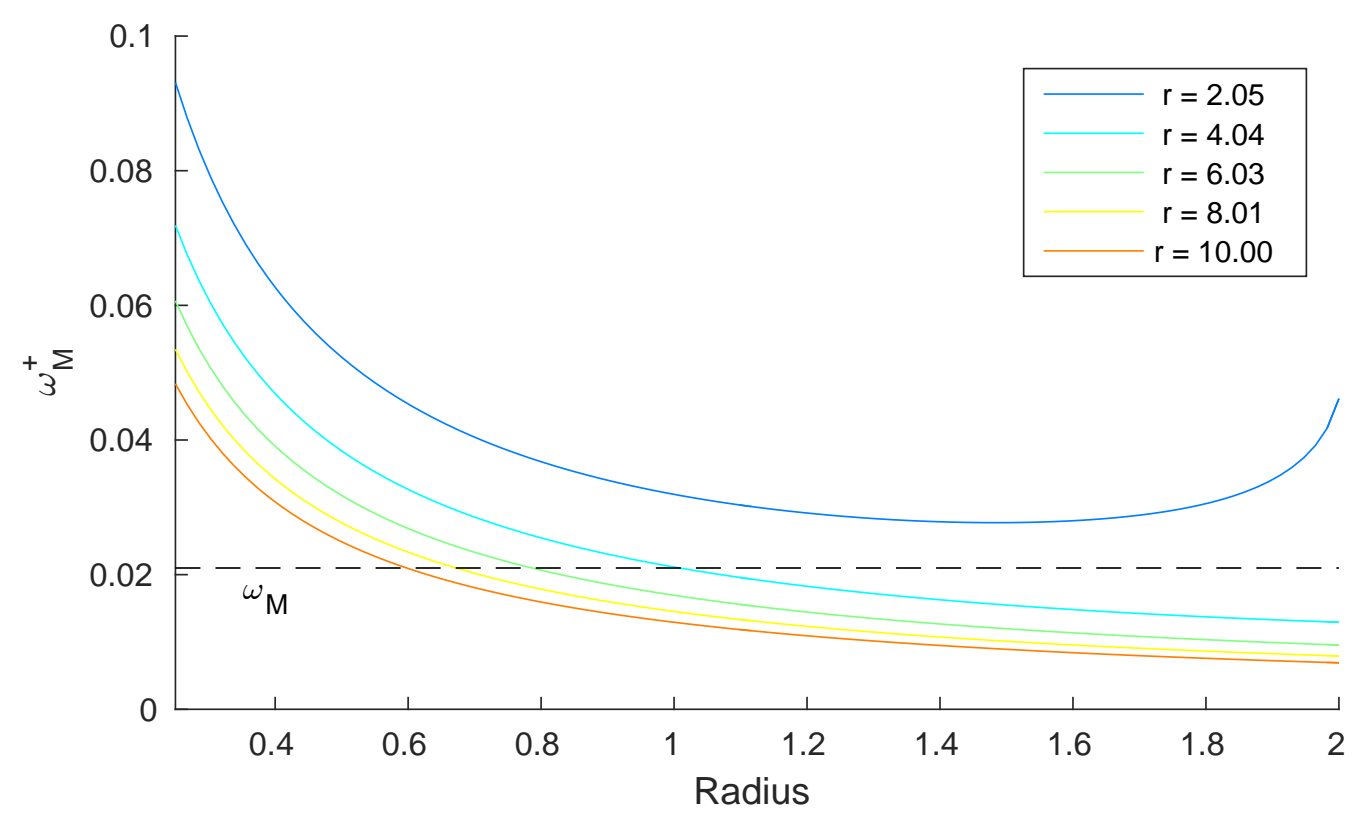

Figure 3: The period is fixed at $a=5$, and the distance from the bubble centers to the reflective plane $\partial D$ is represented by $\beta$. The resonant frequency of a bubble is inversely proportional to its radius. However, in the case of a periodic lattice of bubble near a reflective plane, when the bubble radii $r$ become large enough such that the bubbles are almost touching the plane, the resonant frequency $\omega_{M}^{+}$in fact increases as we further increase the radii. Again, the one bubble resonant frequency $\omega_{M}$, for a bubble of radius 1 in free space, is plotted for reference.

[18] P.P. Ewald. Die berechnung optischer und elektrostatischen gitterpotentiale. Ann. Phys., pages $253-268,1921$.

[19] D. Felbacq and G. Bouchitté. Homogenization of a set of parallel fibres. Waves in random media, 7(2):245-256, 1997.

[20] D. Grieser. The plasmonic eigenvalue problem. Rev. Math. Phys., 26, 1450005, 2014.

[21] D. B. Khismatullin. Resonance frequency of microbubbles: Effect of viscosity. J. Acoust. Soc. Am., 116(3):1463-1473, 2004.

[22] V. Leroy, A. Bretagne, M. Fink, H. Willaime, P. Tabeling, and A. Tourin. Design and characterization of bubble phononic crystals. Appl. Phys. Lett., 95(17):171904, 2009.

[23] V. Leroy, M. Devaud, and J.-C. Bacri. The air bubble: Experiments on an unusual harmonic oscillator. Am. J. Phys., 70(10):1012-1019, 2002.

[24] V. Leroy, A. Strybulevych, M. Lanoy, F. Lemoult, A. Tourin, and J.H. Page. Superabsorption of acoustic waves with bubble metascreens. Phys. Rev. B, 91:020301, 2015.

[25] V. Leroy, A. Strybulevych, M.G. Scanlon, and J.H. Page. Transmission of ultrasound through a single layer of bubbles. Eur. Phys. J. E, 29(1):123-130, 2009. 
[26] J.L. Lions and E. Magenes. Non-homogeneous boundary value problems and applications, volume 1. Springer Berlin Heidelberg, 1972.

[27] M. Minnaert. XVI. On musical air-bubbles and the sounds of running water. The London, Edinburgh, Dublin Philos. Mag. and J. of Sci., 16(104):235-248, 1933.

[28] M. Reed and B. Simon. Methods of Modern Mathematical Physics. Analysis of Operators, volume IV. Academic Press, 1978. 